\title{
Search for heavy Higgs bosons decaying to a top quark pair in proton-proton collisions at $\sqrt{s}=13 \mathrm{TeV}$
}

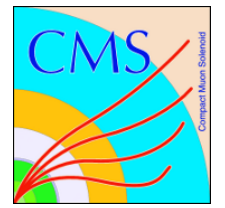

\section{The CMS collaboration}

E-mail: cms-publication-committee-chair@cern.ch

ABSTRACT: A search is presented for additional scalar $(\mathrm{H})$ or pseudoscalar $(\mathrm{A})$ Higgs bosons decaying to a top quark pair in proton-proton collisions at a center-of-mass energy of $13 \mathrm{TeV}$. The data set analyzed corresponds to an integrated luminosity of $35.9 \mathrm{fb}^{-1}$ collected by the CMS experiment at the LHC. Final states with one or two charged leptons are considered. The invariant mass of the reconstructed top quark pair system and variables that are sensitive to the spin of the particles decaying into the top quark pair are used to search for signatures of the $\mathrm{H}$ or $\mathrm{A}$ bosons. The interference with the standard model top quark pair background is taken into account. A moderate signal-like deviation compatible with an A boson with a mass of $400 \mathrm{GeV}$ is observed with a global significance of 1.9 standard deviations. New stringent constraints are reported on the strength of the coupling of the hypothetical bosons to the top quark, with the mass of the bosons ranging from 400 to $750 \mathrm{GeV}$ and their total relative width from 0.5 to $25 \%$. The results of the search are also interpreted in a minimal supersymmetric standard model scenario. Values of $m_{\mathrm{A}}$ from 400 to $700 \mathrm{GeV}$ are probed, and a region with values of $\tan \beta$ below 1.0 to 1.5 , depending on $m_{\mathrm{A}}$, is excluded at $95 \%$ confidence level.

KEYwORDs: Hadron-Hadron scattering (experiments), Higgs physics, Top physics

ArXiv EPrint: 1908.01115 


\section{Contents}

1 Introduction 1

2 The CMS detector and event reconstruction 3

3 Data and simulated event samples 5

4 Data analysis $\quad 7$

$\begin{array}{lll}4.1 & \text { Single-lepton final state } & 7\end{array}$

4.2 Dilepton final state 9

5 Systematic uncertainties $\quad 13$

6 Results $\quad 17$

6.1 Interpolation and extrapolation of signal masses and widths 18

$\begin{array}{ll}6.2 \text { Model-independent interpretation } & 18\end{array}$

$\begin{array}{lll}6.3 & \text { Interpretation in the hMSSM } & 19\end{array}$

$\begin{array}{lll}7 & \text { Summary } & 22\end{array}$

$\begin{array}{ll}\text { The CMS collaboration } & 31\end{array}$

\section{Introduction}

The observation of a Higgs boson with a mass of $125 \mathrm{GeV}$ by the ATLAS and CMS experiments [1-3] was a milestone in particle physics, confirming the existence of a crucial ingredient of the standard model (SM) of particle physics. Multiple extensions of the SM predict new spin-0 states. These include two-Higgs-doublet models (2HDMs) [4], of which the minimal supersymmetric standard model (MSSM) $[5,6]$ is a particular realization, models predicting a new electroweak singlet [7], and other models with a combination of singlet and doublet fields [8]. The additional bosons may also provide a portal to dark matter, by acting as a mediator between SM and dark matter particles $[9,10]$.

The new states introduced in these extensions of the SM may include charged Higgs bosons, $\mathrm{H}^{ \pm}$, scalar (CP-even) neutral $\mathrm{H}$ and h bosons (here h denotes the lighter of the two states), and a pseudoscalar (CP-odd) neutral A boson. For convenience and depending on the context, a common symbol $\Phi$ is used in this paper to represent the $\mathrm{H}$ and $\mathrm{A}$ bosons.

Top quarks play a key role in searches for new physics because of their high mass and large coupling to the SM Higgs boson. Provided that additional Higgs bosons couple to fermions via a Yukawa interaction, the top quark's high mass suggests the size of the coupling to these new bosons to be large as well. Hence, assuming the masses of the 

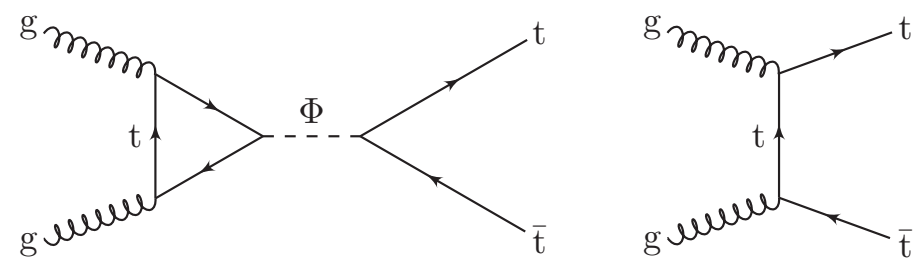

Figure 1. The Feynman diagram for the signal process (left) and an example diagram for the SM production of top quark pairs (right).

new $\Phi$ bosons are sufficiently high, their possible decay to a top quark pair is particularly interesting. Decays of CP-odd Higgs bosons to weak vector bosons, $\mathrm{A} \rightarrow \mathrm{WW}$ and $\mathrm{A} \rightarrow$ $\mathrm{ZZ}$, are forbidden (at tree level) if the CP symmetry is assumed. Such decays are also strongly suppressed for $\mathrm{H}$ bosons in the vicinity of the alignment limit of $2 \mathrm{HDMs}$, in which the properties of the $\mathrm{h}$ boson approach those of the SM Higgs boson [11]. The aligned scenario may naturally occur as a result of a broken symmetry, and such models imply the existence of additional Higgs bosons at relatively low mass $(\lesssim 550 \mathrm{GeV})[12]$. In this paper, however, we do not rely on the assumption of alignment and its naturalness, and probe masses between 400 and $750 \mathrm{GeV}$.

We consider a Yukawa-like coupling between the new spin-0 bosons and the top quark. The corresponding terms in the Lagrangian for the two CP eigenstates read as

$$
\mathcal{L}_{\text {Yukawa, } \mathrm{H}}=-g_{\mathrm{Ht} \overline{\mathrm{t}}} \frac{m_{\mathrm{t}}}{v} \overline{\mathrm{t}} \mathrm{tH}, \quad \mathcal{L}_{\text {Yukawa, } \mathrm{A}}=i g_{\mathrm{Att}} \frac{m_{\mathrm{t}}}{v} \overline{\mathrm{t}} \gamma_{5} \mathrm{tA},
$$

where $m_{\mathrm{t}}$ is the top quark mass, $v$ is the SM Higgs vacuum expectation value, and the strength of the couplings is controlled by real-valued coupling modifiers $g_{\Phi \mathrm{t} \overline{\mathrm{t}}} \geqslant 0$.

A special case of a Type-II 2HDM [4] is the Higgs sector in the hMSSM [13], where the $\mathrm{h}$ boson is identified with the Higgs boson with mass of $125 \mathrm{GeV}$. The hMSSM can be fully described by two tree-level parameters: $\tan \beta$, the ratio of the vacuum expectation values of the two Higgs fields, and $m_{\mathrm{A}}$, the mass of the pseudoscalar boson. The parameter region at low values of $\tan \beta$ is of particular interest, since the coupling of the additional Higgs bosons to top quarks is enhanced in this regime.

We consider the production of new $\Phi$ bosons through the gluon fusion process, with only top quarks in the loop. When the heavy Higgs boson decays into a top quark pair, this mode interferes at the quantum level with the SM production of top quark pairs. Example Feynman diagrams are shown in figure 1. As a consequence, the signal consists of a resonant and an interference component. The resonant component corresponds to the square of the amplitude given by the signal diagram, and results in a Breit-Wigner peak in the distribution of the invariant mass of the $t \overline{\mathrm{t}}$ system, $m_{\mathrm{t} \overline{\mathrm{t}}}$. The interference component may be either destructive or constructive, depending on the phase space region and signal model. The sum of the components may result in a peak-dip structure in the $m_{\mathrm{t} \overline{\mathrm{t}}}$ distribution [14-16]. It is worth noting that the shape and magnitude of the interference depends on the specific signal model, and can be significantly modified by new particles appearing in the loop of the production diagram $[17,18]$. 
Decays of the scalar and pseudoscalar Higgs bosons produce t $\bar{t}$ pairs in the ${ }^{3} P_{0}$ and ${ }^{1} S_{0}$ states respectively [16], while the $\mathrm{SM} \mathrm{gg} \rightarrow \mathrm{t} \overline{\mathrm{t}}$ production results in a mixture of states that changes with the partonic center-of-mass energy. Consequently, the signal and the background exhibit different angular properties, providing an additional handle to distinguish them.

A search for $\mathrm{H}$ or $\mathrm{A}$ bosons decaying to a top quark pair was performed at a centerof-mass energy $\sqrt{s}=8 \mathrm{TeV}$ by the ATLAS experiment [19]. The results were interpreted within the context of a Type-II 2HDM. The CMS experiment performed a search for top quark associated production of an $\mathrm{H}$ or $\mathrm{A}$ boson decaying to a top quark pair at $\sqrt{s}=13 \mathrm{TeV}$ [20]. The ATLAS and CMS collaborations also searched for spin-1 and spin2 resonances decaying to a top quark pair [21, 22], generally probing very high masses and Lorentz-boosted topologies, and without considering quantum interference with SM top quark pair production. In addition, both collaborations performed searches for $\mathrm{H}^{ \pm}$ decaying to a top and a bottom quark [23, 24], which are also sensitive to the region of low $\tan \beta$ in the hMSSM parameter space.

This paper describes a search for $\mathrm{H}$ or $\mathrm{A}$ bosons decaying to a top quark pair in proton-proton collisions at $\sqrt{s}=13 \mathrm{TeV}$ using the CMS detector at the CERN LHC. The data set analyzed corresponds to an integrated luminosity of about $35.9 \mathrm{fb}^{-1}$, collected in 2016. Events are selected in which the top quark pair decays into a final state with one or two leptons, where a lepton refers to an electron or a muon throughout this article. This analysis exploits both $m_{\mathrm{t} \overline{\mathrm{t}}}$ and angular variables sensitive to the spin of the heavy Higgs bosons. Constraints on the coupling modifier $g_{\Phi t \bar{t}}$ are derived as a function of the boson mass and width. The results are also interpreted in the hMSSM context, putting constraints in the $\left(m_{\mathrm{A}}, \tan \beta\right)$ plane.

\section{The CMS detector and event reconstruction}

The central feature of the CMS apparatus is a superconducting solenoid of $6 \mathrm{~m}$ internal diameter, providing a magnetic field of $3.8 \mathrm{~T}$. Within the solenoid volume are a silicon pixel and strip tracker, a lead tungstate crystal electromagnetic calorimeter (ECAL), and a brass and scintillator hadron calorimeter (HCAL), each composed of a barrel and two endcap sections. Forward calorimeters extend the pseudorapidity coverage provided by the barrel and endcap detectors. Muons are detected in gas-ionization chambers embedded in the steel flux-return yoke outside the solenoid. A more detailed description of the CMS detector, together with a definition of the coordinate system used and the relevant kinematic variables, can be found in ref. [25].

The reconstructed vertex with the largest value of summed physics-object $p_{\mathrm{T}}^{2}$ is taken to be the primary pp interaction vertex. The physics objects are the jets, clustered using the jet finding algorithm $[26,27]$ with the tracks assigned to the vertex as inputs, and the associated missing transverse momentum, taken as the negative vector sum of the $p_{\mathrm{T}}$ of those jets.

The particle-flow (PF) algorithm [28] aims to reconstruct and identify each individual particle in an event, with an optimized combination of information from the various ele- 
ments of the CMS detector. The reconstructed particles are referred to as PF candidates in the following. The energy of photons is obtained from the ECAL measurement. The energy of electrons is determined from a combination of the electron momentum at the primary interaction vertex as determined by the tracker, the energy of the corresponding ECAL cluster, and the energy sum of all bremsstrahlung photons spatially compatible with originating from the electron track. The energy of muons is obtained from the curvature of the corresponding track. The energy of charged hadrons is determined from a combination of their momentum measured in the tracker and the matching ECAL and HCAL energy deposits, corrected for zero-suppression effects and for the response function of the calorimeters to hadronic showers. Finally, the energy of neutral hadrons is obtained from the corresponding corrected ECAL and HCAL energies.

Jets are reconstructed from the PF candidates with the infrared and collinear safe anti- $k_{\mathrm{T}}$ algorithm [27] operated with a distance parameter $R=0.4$. Jet momentum is determined as the vectorial sum of all particle momenta in the jet, and is found from simulation to be, on average, within 5 to $10 \%$ of the true momentum over the whole $p_{\mathrm{T}}$ spectrum and detector acceptance. Additional pp interactions within the same or nearby bunch crossings, referred to as pileup, can contribute additional tracks and calorimetric energy depositions to the jet momentum. To mitigate this effect, tracks identified to be originating from pileup vertices are discarded, and an offset correction is applied to correct for remaining contributions. Jet momentum corrections are derived from simulation to bring the measured response of jets to that of particle level jets on average. In situ measurements of the momentum balance in dijet, photon + jet, $\mathrm{Z}+$ jet, and quantum chromodynamics (QCD) multijet events (consisting uniquely of jets produced through the strong interaction), are used to account for any residual differences in jet $p_{\mathrm{T}}$ scale in data and simulation [29]. The relative jet $p_{\mathrm{T}}$ resolution amounts typically to $15-20 \%$ at $30 \mathrm{GeV}$, $10 \%$ at $100 \mathrm{GeV}$, and $5 \%$ at $1 \mathrm{TeV}$. Additional selection criteria are applied to each jet to remove jets potentially dominated by anomalous contributions from various subdetector components or reconstruction failures [30].

Jets originating from b quarks are identified with the cMVAv2 algorithm [31], combining six different $\mathrm{b}$ jet discriminators, which exploit displaced track and secondary vertex information. The collection of b-tagged jets is defined by an operating point that corresponds to a b tagging efficiency of about $66 \%$ for b jets, $13 \%$ for c jets, and a misidentification probability ("mistag rate") of about $1 \%$ for light-flavor jets. Differences between data and simulation in the $\mathrm{b}$ tagging efficiency and mistag rate are accounted for by scale factors that depend on the jet $p_{\mathrm{T}}$ and $\eta$.

The electron momentum is estimated by combining the energy measurement in the ECAL with the momentum measurement in the tracker. The momentum resolution for electrons with $p_{\mathrm{T}} \approx 45 \mathrm{GeV}$ from $\mathrm{Z} \rightarrow$ ee decays ranges from $1.7 \%$ for nonshowering electrons in the barrel region to $4.5 \%$ for showering electrons in the endcaps [32]. Muons are measured in the pseudorapidity range $|\eta|<2.4$, with detection planes made using three technologies: drift tubes, cathode strip chambers, and resistive plate chambers. Matching muons to tracks measured in the silicon tracker results in a relative transverse momentum resolution for muons with $20<p_{\mathrm{T}}<100 \mathrm{GeV}$ of $1.3-2.0 \%$ in the barrel and better than $6 \%$ 
in the endcaps. The $p_{\mathrm{T}}$ resolution in the barrel is better than $10 \%$ for muons with $p_{\mathrm{T}}$ up to $1 \mathrm{TeV}$ [33]. Simulation-to-data scale factors that depend on the lepton $p_{\mathrm{T}}$ and $\eta$ are used to correct for small differences in lepton trigger, identification, and isolation efficiency.

We define tight and loose collections of electron and muon candidates, corresponding to the stringency of the lepton identification criteria. For electrons, an updated version of the criteria from ref. [32] is utilized, while the muon identification is as described in ref. [33]. Tight and loose electrons are furthermore required to satisfy $p_{\mathrm{T}}>20 \mathrm{GeV}$, while tight (loose) muons have $p_{\mathrm{T}}>20(10) \mathrm{GeV}$. The relative lepton isolation, $I_{\text {rel }}$, is calculated as the sum of the transverse momenta of charged-hadron, neutral-hadron, and photon PF candidates, inside a cone of $\Delta R=\sqrt{(\Delta \eta)^{2}+(\Delta \phi)^{2}}=0.4$ around the lepton, divided by the lepton $p_{\mathrm{T}}$. An estimated contribution from pileup is subtracted in this calculation. Tight electrons must satisfy $I_{\text {rel }} \lesssim 0.06$, while loose ones are required to have $I_{\text {rel }} \lesssim 0.18(0.16)$ in the barrel (endcap) region. Tight (loose) muon candidates must satisfy $I_{\text {rel }}<0.15(0.25)$.

The variable $p_{\mathrm{T}}^{\text {miss }}$, referred to as the missing transverse momentum [34], is defined as the magnitude of the missing transverse momentum vector $\vec{p}_{\mathrm{T}}^{\text {miss }}$, which is the projection on the plane perpendicular to the beams of the negative vector sum of the momenta of all reconstructed $\mathrm{PF}$ candidates in the event. The energy corrections applied to the jets are propagated to the $p_{\mathrm{T}}^{\text {miss }}$ calculation.

\section{Data and simulated event samples}

This analysis is performed on a pp collision data set recorded during 2016, at a centerof-mass energy of $13 \mathrm{TeV}$. The total integrated luminosity of the collected data sample is $35.9 \pm 0.9 \mathrm{fb}^{-1}[35]$.

The single-electron (single-muon) data sample is selected with triggers [36] based on the presence of an isolated electron (muon). The dielectron, electron-muon, and dimuon data samples are selected with triggers that require the presence of two leptons of the corresponding flavors. In order to increase the selection efficiency for dilepton events in which the subleading lepton has a relatively low $p_{\mathrm{T}}$, all the dilepton samples are further extended with events that pass the single-lepton but not the dilepton triggers.

In order to compare the collected data to theoretical predictions, Monte Carlo (MC) samples are produced with events simulating the $\Phi \rightarrow \mathrm{t} \overline{\mathrm{t}}$ signal and SM background processes. The signal is simulated at leading order (LO) accuracy in perturbative QCD using a custom model in the MADGRAPH5_aMC@NLO 2.5.1 event generator [37] that implements the top quark loop of the gluon fusion production via an effective coupling between the new boson and gluons [38]. The generator employs the NNPDF3.0 parton distribution functions (PDFs) [39], and is interfaced with PYTHIA 8.212 [40] for fragmentation and hadronization, with the CUETP8M1 underlying event tune [41, 42]. Signal event samples are produced for (pseudo)scalar boson masses of 400,500,600, and $750 \mathrm{GeV}$, with relative total decay widths $\Gamma_{\Phi} / m_{\Phi}$ of $2.5,5,10$, and $25 \%$ for each mass scenario. The factorization and renormalization scales, $\mu_{\mathrm{F}}$ and $\mu_{\mathrm{R}}$, are set on an event-by-event basis to $m_{\mathrm{t} \overline{\mathrm{t}}} / 2$, following the choice in ref. [43]. The top quarks from the heavy Higgs boson decay are decayed in MAdGraph5_amC@NLO, preserving their spin correlations. Samples are generated for 
events corresponding to the resonant heavy boson signal, and for events corresponding to interference terms in the matrix element calculation between the signal and SM t $\overline{\mathrm{t}}$ background. Events in the interference samples can receive negative weights, which reflects the sign of the corresponding part of the squared matrix element in the presence of a destructive interference. Since the heavy Higgs boson is produced via gluon fusion with a top quark loop, the coupling between the boson and the top quark appears twice in the matrix element. As a result, events originating from the resonance (interference) matrix element terms correspond to a cross section that is proportional to $g_{\Phi \mathrm{t} \overline{\mathrm{t}}}^{4}\left(g_{\Phi \mathrm{t} \overline{\mathrm{t}}}^{2}\right)$.

We calculate the next-to-next-to-LO (NNLO) cross sections for the resonant part of a given signal using the SusHi 1.6.1 program [44]. The ratio of the NNLO cross section over the LO cross section computed with MADGRAPH5_amC@NLO determines the $K$ factor, typically of size $\approx 2$, applied to the resonant part of the signal. The $K$ factors applied to the interference component of the signal are obtained as the geometric mean of the

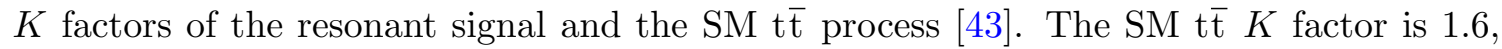
calculated as the ratio between the NNLO cross section used for the simulated t $\overline{\mathrm{t}}$ sample, as described below, and the LO cross section obtained in a similar setup. The $K$ factors for the resonant part of the signal and the interference are applied throughout this analysis. In the hMSSM interpretation, we also use the 2 HDMC program [45] to calculate, for given $m_{\mathrm{A}}$ and $\tan \beta$, the mass of the $\mathrm{H}$ boson and the widths of both heavy Higgs bosons, as well as other MSSM parameters.

The main SM background contribution in this analysis originates from t $\overline{\mathrm{t}}$ production. Other background events originate from single top quark production, single boson production (Drell-Yan $\mathrm{Z} / \gamma^{*}+$ jets and $\mathrm{W}+$ jets), diboson processes (WW, WZ, and ZZ), $\mathrm{t} \overline{\mathrm{t}}$ production in association with a $\mathrm{Z}$ or $\mathrm{W}$ boson (commonly referred to as $\mathrm{t} \overline{\mathrm{t}} \mathrm{V}$ ), and QCD multijet processes.

The t $\overline{\mathrm{t}}$ process is simulated to next-to-LO (NLO) using the POWHEG v2 generator [4649], assuming a top quark mass of $172.5 \mathrm{GeV}$. The factorization and renormalization scales are set to $\sqrt{m_{\mathrm{t}}^{2}+p_{\mathrm{T}, \mathrm{t}}^{2}}$, where $m_{\mathrm{t}}$ and $p_{\mathrm{T}, \mathrm{t}}$ are the mass and the transverse momentum of the top quarks in the underlying Born-level configuration. The NNPDF3.0 PDF set is used, and the events are passed to PYTHIA with the CUETP8M2T4 event tune [50]. The predicted $t \overline{\mathrm{t}}$ production cross section is $831.8_{-29.2}^{+19.8}$ (scale) $\pm 35.1\left(\mathrm{PDF}+\alpha_{S}\right) \mathrm{pb}$, as calculated with the TOP ++2.0 program to NNLO in perturbative QCD, including softgluon resummation to next-to-next-to-leading logarithmic (NNLL) order (as discussed in ref. [51] and references therein), and assuming a top quark mass of $172.5 \mathrm{GeV}$. The first uncertainty comes from the independent variation of the factorization and renormalization scales, while the second one is associated to variations in the PDF and strong coupling $\alpha_{S}$, following the PDF4LHC prescription with the MSTW2008 68\% confidence level NNLO, CT10 NNLO, and NNPDF2.3 5f FFN PDF sets (as discussed in ref. [52] and references therein, and refs. [53-55]). The modeling of SM t $\overline{\mathrm{t}}$ production in POWHEG is known to predict a harder $p_{\mathrm{T}}$ spectrum of the top quarks than observed in the data. An empirical reweighting for top quark pairs based on the $p_{\mathrm{T}}$ spectrum of generator-level top quarks is applied to obtain a better agreement with the measured differential t $\bar{t}$ cross section $[56,57]$. 
The single top quark production processes, via the $t, \mathrm{tW}$, and $s$ channels, are generated at NLO using POWheg v2, POWheg, and MAdGraPh5_amC@NLO, respectively. The samples are normalized using the NLO cross section predictions for the $t$ and $s$ channels [58, 59], and approximate NNLO prediction for the tW channel [60]. The $\mathrm{Z} / \gamma^{*}+$ jets, $\mathrm{W}+$ jets, and $t \bar{t} \mathrm{~V}$ event samples are generated using MADGRAPH5_aMC@NLO. For the single boson production processes, events with up to four additional partons are generated at LO, and the MLM matching scheme [61] is employed to combine the different parton multiplicities. The single-boson production cross sections are calculated at NNLO $[62,63]$. In the dilepton analysis, the normalization of the $\mathrm{Z} / \gamma^{*}+$ jets contribution is determined from a control

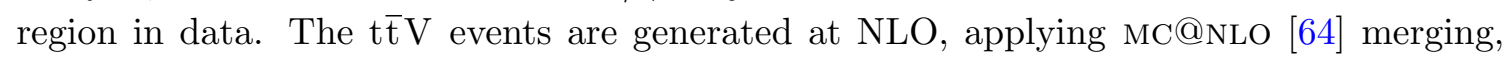
and are normalized using NLO cross section predictions. Events simulating the diboson processes are generated using PYTHIA and normalized to the respective NNLO (for WW production) [65] or NLO (for WZ and ZZ production) [66] cross sections. The modeling of QCD multijet events is obtained from a control region in data, but events simulated with PYTHIA are used to validate the modeling. All events are interfaced with PYTHIA for the fragmentation and hadronization, using the CUETP8M2T4 (single top processes) or CUETP8M1 (others) tunes.

The simulated events are processed through the CMS detector simulation based on the Geant4 program [67]. Pileup events generated with Pythia are overlaid in all samples, to simulate additional interactions in the same bunch crossing. The simulated events are weighted to reproduce the distribution of the number of pileup interactions observed in data. On average, there are 23 collisions per bunch crossing.

\section{Data analysis}

We search for heavy Higgs bosons decaying into a top quark pair, in final states with either one or two leptons, where the lepton is either an electron or a muon. The analysis strategy for single-lepton final states differs from the one for dilepton final states, due to differences in the event selection, SM background composition, kinematic top quark pair reconstruction, and definition of observables that discriminate between the SM background and the signal.

\subsection{Single-lepton final state}

In the single-lepton channel we aim to select events originating from top quark pair decays to a leptonically decaying $\mathrm{W}$ boson and a hadronically decaying $\mathrm{W}$ boson. The targeted topology is therefore $\mathrm{t} \overline{\mathrm{t}} \rightarrow \ell^{+} \nu \mathrm{b} q \overline{\mathrm{q}} \overline{\mathrm{b}}$, where $\ell$ denotes an electron or a muon and the leptonic and hadronic $\mathrm{W}$ boson decays may be swapped. Events in the single-electron (single-muon) channel in data and simulation are required to pass a single-electron (singlemuon) trigger, as explained in section 3. Selected events must have exactly one tight electron (muon) with $p_{\mathrm{T}}>30(26) \mathrm{GeV}$ and $\left|\eta_{\mathrm{SC}}\right|<2.5(|\eta|<2.4)$, where $\eta_{\mathrm{SC}}$ is the pseudorapidity of the ECAL supercluster associated with the electron [32]. To suppress the contribution from $\mathrm{Z} / \gamma^{*}+$ jets and other processes in which multiple prompt leptons are produced, an event is rejected if an additional loose electron or loose muon is found. 
This also ensures orthogonality to the event selection of the dilepton analysis outlined in section 4.2. An event must contain at least four jets with $p_{\mathrm{T}}>20 \mathrm{GeV}$ and $|\eta|<$ 2.4 , at least two of which are required to be $\mathrm{b}$ tagged. To further suppress the QCD multijet background, only events with $m_{\mathrm{T}}^{\mathrm{W}}>50 \mathrm{GeV}$ are selected. The transverse mass variable is defined as $m_{\mathrm{T}}^{\mathrm{W}}=\sqrt{2 p_{\mathrm{T}}^{\ell} p_{\mathrm{T}}^{\text {miss }}\left[1-\cos \Delta \phi\left(\vec{p}_{\mathrm{T}}^{\ell}, \vec{p}_{\mathrm{T}}^{\text {miss }}\right)\right]}$, with $\vec{p}_{\mathrm{T}}^{\ell}$ being the transverse momentum of the only tight electron or muon in the event.

Each event that passes the selection described above is reconstructed under the assumption that it has been produced in the process $t \bar{t} \rightarrow \ell \nu b \bar{b} q \bar{q}^{\prime}$. The $t \bar{t}$ system is reconstructed using an approach similar to the one adopted in ref. [56]. All possible ways to assign four reconstructed jets to the four quarks in the final state are considered. To reduce the number of combinations, each of the two b quarks is required to be associated to a b-tagged jet. For each considered choice of a jet assigned to a b quark stemming from the semileptonically decaying top quark, an attempt is made to reconstruct the transverse momentum of the neutrino, $\vec{p}_{\mathrm{T}}^{\nu}[68]$. This is achieved by imposing a constraint on the mass of the semileptonically decaying top quark and on the mass of the leptonically decaying $\mathrm{W}$ boson, and choosing the unique solution of the neutrino momentum that minimizes the distance $D_{\nu}=\left|\vec{p}_{\mathrm{T}}^{\nu}-\vec{p}_{\mathrm{T}}^{\text {miss }}\right|$. Next, a likelihood function is constructed from the probability density function of the minimal value of $D_{\nu}$, and the two-dimensional (2D) probability density function of the reconstructed mass of the top quark and the $\mathrm{W}$ boson in the hadronic side of the $t \bar{t}$ decay. The jet assignment with the largest value of this likelihood is chosen to reconstruct the $t \overline{\mathrm{t}}$ system.

The performance of the $t \bar{t}$ reconstruction algorithm is studied using SM t $\bar{t}$ simulation, considering only events with the targeted decays at the generator level, $t \overline{\mathrm{t}} \rightarrow \ell \nu \mathrm{b} \overline{\mathrm{b}} \mathrm{q} \overline{\mathrm{q}}^{\prime}$. A correct jet-quark assignment exists for $44 \%$ of such events that pass the event selection. For those events where a correct assignment exists and a solution can be found for the neutrino momentum, the probability that all four jets are correctly assigned to the quarks varies from around 60 to $80 \%$, depending on the value of the invariant mass of the generator-level top quark pair, $m_{\mathrm{t} \overline{\mathrm{t}}}^{\mathrm{gen}}$. The relative $m_{\mathrm{t} \overline{\mathrm{t}}}$ resolution, as computed with all selected events with targeted decays, changes from about 17 to $21 \%$, depending on $m_{\mathrm{t} \overline{\mathrm{t}}}^{\text {gen }}$.

The $t \bar{t}$ reconstruction results in a solution in about $85 \%$ of observed events, and only the events with a solution are considered for further analysis. The search for the $\Phi \rightarrow t \bar{t}$ signal is performed using two observables. The first one is the invariant mass $m_{\mathrm{t} \overline{\mathrm{t}}}$, as obtained from the $t \bar{t}$ reconstruction algorithm, and probes the mass of the heavy (pseudo)scalar boson. The second observable is $\left|\cos \theta_{\mathrm{t}_{\ell}}^{*}\right|$, where $\theta_{\mathrm{t}_{\ell}}^{*}$ denotes the angle between the momentum of the semileptonically decaying top quark in the t $\bar{t}$ rest frame and the momentum of the $t \bar{t}$ system in the laboratory frame, as illustrated in figure 2. In a $\Phi \rightarrow t \bar{t}$ signal process, the heavy Higgs boson would decay into top quarks isotropically, resulting in a flat distribution of $\cos \theta_{\mathrm{t}_{\ell}}^{*}$ at the generator level. The SM t $\overline{\mathrm{t}}$ production, on the other hand, yields a distribution peaking at \pm 1 . As a result, the distribution of $\left|\cos \theta_{t_{\ell}}^{*}\right|$ is relatively enriched in signal events towards $\left|\cos \theta_{\mathrm{t}_{\ell}}^{*}\right|=0$.

The QCD multijet background is estimated from dedicated control regions in the data, independently in the electron and muon channels. The total event yield for this background 


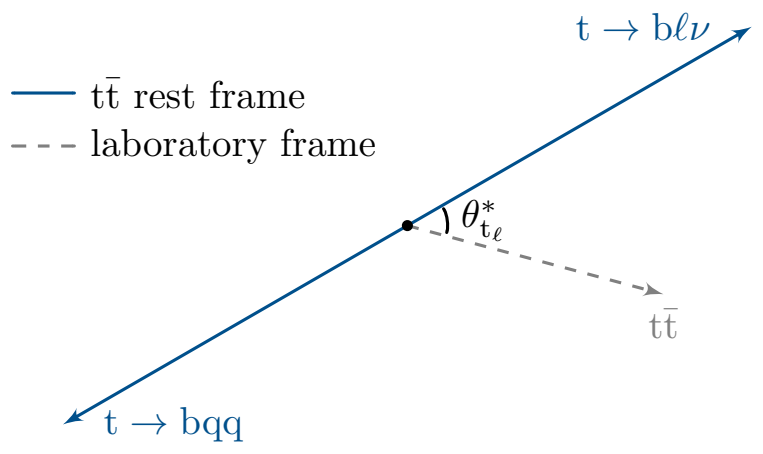

Figure 2. Definition of angle $\theta_{\mathrm{t}_{\ell}}^{*}$ used in the single-lepton final state. Momenta in different coordinate systems are depicted with arrows of different colors and styles.

is obtained from data using a variant of the ABCD method [69]. Four regions are defined, based on the relative isolation of the lepton (smaller or greater than the isolation threshold imposed on the tight lepton), and the $m_{\mathrm{T}}^{\mathrm{W}}$ variable (smaller or greater than $50 \mathrm{GeV}$ ). The three regions complementary to the signal region are relatively enriched in the multijet background. The overall rate of QCD multijet background in the signal region is estimated with a simultaneous fit to the numbers of events observed in the four regions, exploiting the factorization of the distribution of this background in $\left(m_{\mathrm{T}}^{\mathrm{W}}, I_{\mathrm{rel}}\right)$. The shape of the multijet distribution of the observables $m_{\mathrm{t} \overline{\mathrm{t}}}$ and $\left|\cos \theta_{\mathrm{t}_{\ell}}^{*}\right|$ is determined from data using events with an inverted lepton isolation selection applied, after subtracting the contributions from other backgrounds.

The data and the expected SM background yields are shown in table 1, for selected events that have a solution of the t $\bar{t}$ reconstruction algorithm. The background predictions are computed with the help of a maximum likelihood fit to the data using the backgroundonly version of the full statistical model that will be described in section 6 . The uncertainties obtained in this fit, referred to as post-fit uncertainties, are reported.

The observed and post-fit predicted distributions of $m_{\mathrm{t} \overline{\mathrm{t}}}$ in different $\left|\cos \theta_{\mathrm{t}_{\ell}}^{*}\right|$ regions are shown in figure 3. The impact of the signal process (including the interference) for the best-fit signal hypothesis, which will be discussed in section 6.2 , is shown in the lower panels. It demonstrates the characteristic peak-dip lineshape discussed in section 1. For this benchmark and also in general, the contributions from both the resonant part and the interference are important. The relative importance of the latter increases with the total width or as the coupling modifier decreases.

\subsection{Dilepton final state}

In the dilepton channel we aim to select signal events where both top quarks decay to a leptonically decaying $\mathrm{W}$ boson. Hence, the targeted decay topology is $\mathrm{t} \overline{\mathrm{t}} \rightarrow \ell^{+} \nu \mathrm{b} \ell^{-} \bar{\nu} \overline{\mathrm{b}}$. Events in the dielectron (ee), electron-muon $(\mathrm{e} \mu)$, and dimuon $(\mu \mu)$ channel in data and simulation are required to pass a dielectron, electron-muon, and dimuon trigger, respectively, or a single-lepton trigger, as explained in section 3. The subsequent event selection closely follows ref. [70]. Events are required to contain exactly one pair of oppositely charged 


\begin{tabular}{|c|c|c|}
\hline Process & \multicolumn{2}{|c|}{ Event yield } \\
\hline $\begin{array}{l}\text { Observed } \\
\text { Total background }\end{array}$ & $\begin{array}{c}274821 \\
\left(274.8_{-0.9}^{+0.8}\right) \times 10^{3}\end{array}$ & $\begin{array}{c}416254 \\
\left(416.3_{-1.2}^{+1.1}\right) \times 10^{3}\end{array}$ \\
\hline & \multicolumn{2}{|c|}{ Fraction w.r.t. total background } \\
\hline $\mathrm{t} \overline{\mathrm{t}}$ & $91.9 \%$ & $92.1 \%$ \\
\hline Single top quark & $3.9 \%$ & $4.0 \%$ \\
\hline $\mathrm{W}+$ jets & $1.9 \%$ & $2.1 \%$ \\
\hline $\mathrm{Z} / \gamma^{*}+$ jets & $0.4 \%$ & $0.3 \%$ \\
\hline $\mathrm{t} \overline{\mathrm{t} V}$ & $0.2 \%$ & $0.2 \%$ \\
\hline Diboson & $0.1 \%$ & $0.1 \%$ \\
\hline QCD multijet & $1.5 \%$ & $1.0 \%$ \\
\hline
\end{tabular}

Table 1. Event yields and composition of SM background in the single-electron and single-muon channels. Expected yields are computed after the background-only fit to the data as explained in the text.

tight leptons, in which the leading (subleading) lepton has $p_{\mathrm{T}}>25(20) \mathrm{GeV}$. Events are rejected if they contain additional tight electrons or additional muons that satisfy the tight identification criteria with the exception of a looser selection on isolation, $I_{\text {rel }}<0.25$. The selected dilepton pair is further required to have an invariant mass of at least $20 \mathrm{GeV}$, to suppress events from low-mass dilepton resonances. In the ee and $\mu \mu$ channels, events are rejected if they contain a dilepton pair consistent with a decay of a $\mathrm{Z}$ boson, namely, if they have an invariant mass in the range $76-106 \mathrm{GeV}$. Each event must have at least two jets with $p_{\mathrm{T}}>30 \mathrm{GeV}$ and $|\eta|<2.4$. Additional jets with $p_{\mathrm{T}}>20 \mathrm{GeV}$ and $|\eta|<2.4$ in the event are also considered for further analysis. At least one of the jets is required to be b tagged. In the ee and $\mu \mu$ channels, the $p_{\mathrm{T}}^{\text {miss }}$ must exceed $40 \mathrm{GeV}$ to further suppress $\mathrm{Z} / \gamma^{*}$ background events.

The contribution from the $\mathrm{Z} / \gamma^{*}+$ jets background process to the selected event yield is estimated using control regions in data, following the procedure described in ref. [71]. The yield outside the $\mathrm{Z}$ boson mass window is estimated based on the observed event yield within the window, using the knowledge from simulation of the ratio of $\mathrm{Z} / \gamma^{*}+$ jets inside and outside of the mass window. In this estimation, the non- $\mathrm{Z} / \gamma^{*}+$ jets background contribution within the $\mathrm{Z}$ boson mass window is taken from the $\mathrm{e} \mu$ channel, where the $\mathrm{Z} / \gamma^{*}+$ jets contribution is negligible, and corrected for lepton reconstruction effects before being subtracted from the observed number of events in the $\mathrm{Z}$ boson mass window. Using this method, the theoretically predicted $\mathrm{Z} / \gamma^{*}+$ jets event yield is scaled by a factor 1.22 , 1.20 , and 1.19 in the ee, $\mathrm{e} \mu$, and $\mu \mu$ channels respectively.

Each event that passes the selection described above is reconstructed under the assumption that it has been produced in a process $\mathrm{t} \overline{\mathrm{t}} \rightarrow \ell^{+} \nu \mathrm{b} \ell^{-} \bar{\nu} \overline{\mathrm{b}}$. A kinematic recon-

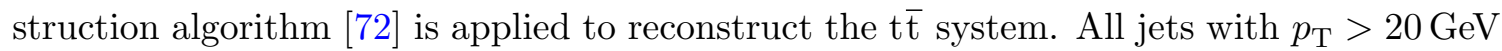
are considered in the reconstruction of the $t \bar{t}$ system. Given an assignment of jets and 

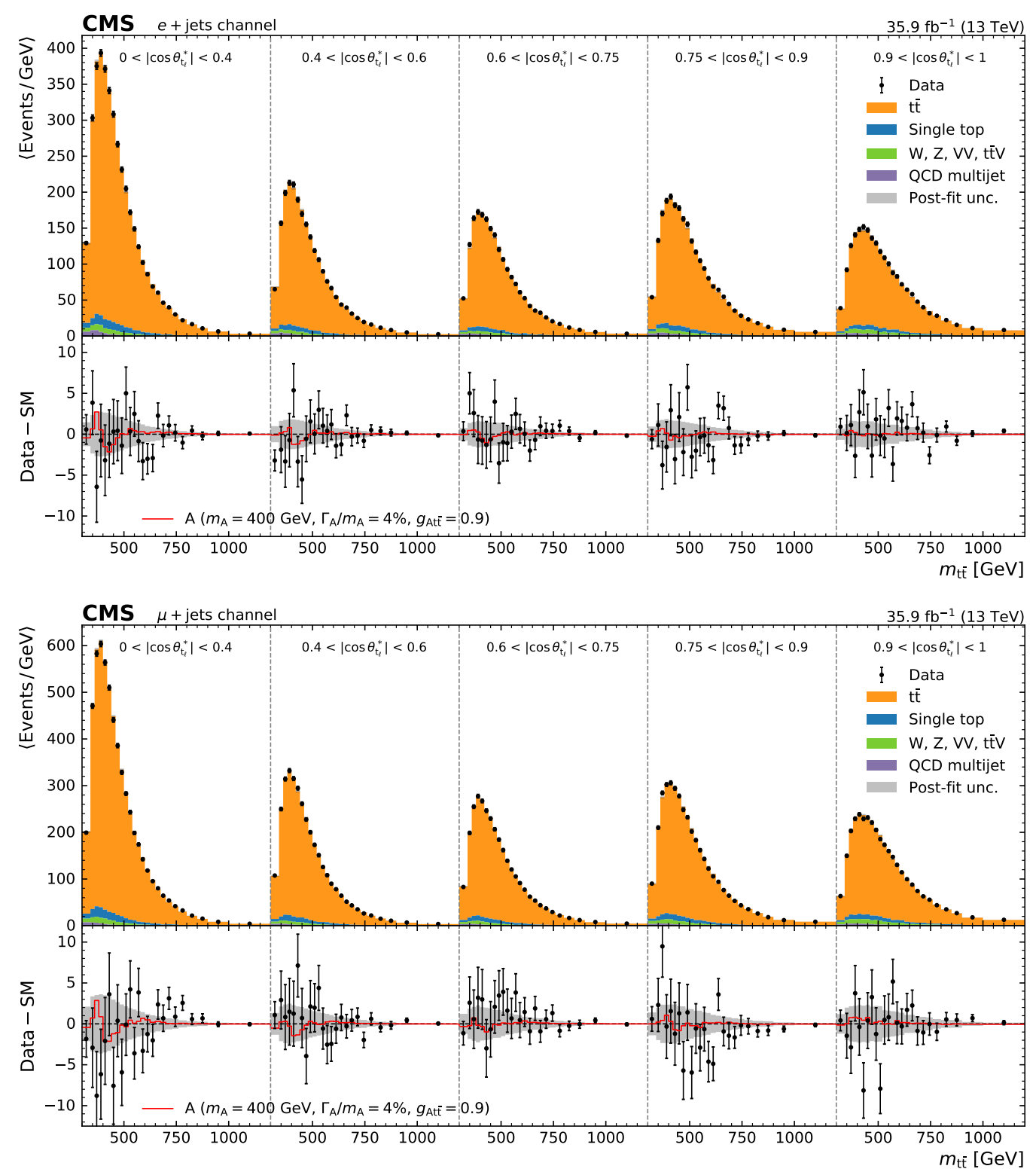

Figure 3. Observed and expected distributions of $m_{\mathrm{t}}$ in different $\left|\cos \theta_{\mathrm{t}_{\ell}}^{*}\right|$ regions in the e + jets (upper) and $\mu+$ jets (lower) channels. The expected distributions have been obtained with a background-only fit to the data, and an approximate post-fit uncertainty is shown with a gray band. The impact of the best-fit signal is included in the lower panels for illustration.

leptons to the underlying expected $t \bar{t}$ decay products, a system of equations is constructed that imposes constraints on the reconstructed $\mathrm{W}$ boson mass and the reconstructed top quark and antiquark masses. The transverse momentum imbalance, represented by $p_{\mathrm{T}}^{\text {miss }}$, is assumed to originate solely from the two neutrinos. Detector resolution effects are taken into account by sampling both the measured energy and the direction of the leptons and $\mathrm{b}$ jet candidates within their respective experimental resolutions. For each sampling, the solution of the equations that results in the smallest $m_{\mathrm{t} \overline{\mathrm{t}}}$ is chosen. Per event, 100 sam- 


\begin{tabular}{|lc|}
\hline Process & Event yield \\
\hline Observed & 230233 \\
Total background & $(231.1 \pm 0.8) \times 10^{3}$ \\
\hline & Fraction w. r. t. total background \\
\hline $\mathrm{t} \overline{\mathrm{t}}$ & $93.3 \%$ \\
Single top quark & $3.3 \%$ \\
$\mathrm{Z} / \gamma^{*}+$ jets & $2.9 \%$ \\
$\mathrm{t} \overline{\mathrm{t}} \mathrm{V}$ & $0.3 \%$ \\
Diboson & $0.1 \%$ \\
\hline
\end{tabular}

Table 2. Event yields and composition of SM background in the dilepton channel. Expected yields are computed in the same way as in table 1.

plings are performed, and each is assigned a weight based on the probability density of the invariant mass of the lepton and $\mathrm{b}$ jet from the top quark decay. The kinematic properties of the top quark and antiquark are obtained as a weighted average over all samplings. Finally, the assignment of jets resulting in the maximum sum of weights over all samplings is chosen, and preference is given to a jet assignment that contains two b-tagged jets.

The performance of the $t \overline{\mathrm{t}}$ reconstruction algorithm is studied using simulated SM t $\overline{\mathrm{t}}$ events with targeted decays at the generator level, $\mathrm{t} \overline{\mathrm{t}} \rightarrow \ell^{+} \nu \mathrm{b} \ell^{-} \bar{\nu} \overline{\mathrm{b}}$. In $75 \%$ of the selected events the two generator-level jets are within the acceptance. For those events for which the algorithm has a solution, the probability to correctly match both $b$ jets as chosen by the algorithm to jets originating from b quarks from the top quark decays is 55 to $85 \%$, depending on the value of $m_{\mathrm{t} \overline{\mathrm{t}}}^{\text {gen }}$. The $m_{\mathrm{t} \overline{\mathrm{t}}}$ resolution, computed using all selected SM t $\overline{\mathrm{t}}$ events with targeted decays, ranges from 20 to $28 \%$, depending on $m_{\mathrm{t} \overline{\mathrm{t}}}^{\mathrm{gen}}$.

The events for which the $t \bar{t}$ reconstruction results in a solution, which is the case in about $95 \%$ of observed events, are considered for further analysis. The resulting event yields for data and SM background expectations are shown in table 2.

The search for the $\Phi \rightarrow t \bar{t}$ signal is performed using two observables. The first one is the invariant mass $m_{\mathrm{t} \overline{\mathrm{t}}}$, obtained from the $\mathrm{t} \overline{\mathrm{t}}$ reconstruction algorithm. The second observable is a spin correlation variable constructed from the charged leptons in the event. Charged leptons have the highest spin analyzing power amongst the top quark decay products [73], and their properties can be measured precisely. The chosen variable is the cosine of the angle between the charged lepton momenta in their respective helicity frames, and is denoted by $c_{\text {hel }}$. The four-momenta of the leptons in their helicity frames are obtained by first boosting the leptons into the $t \bar{t}$ rest frame and then boosting them along their parent top quark or antiquark directions in this frame. The distribution of $c_{\text {hel }}$ is sensitive to the spin and $\mathrm{CP}$ state of the $\mathrm{t} \overline{\mathrm{t}}$ system. At the generator level and with no requirements on acceptance, the distribution is linear in shape; its slope is maximally positive for the A resonance and is mildly negative for the $\mathrm{H}$ resonance. On the other hand, the slope for the $\mathrm{SM} t \overline{\mathrm{t}}$ production, integrated over the entire $m_{\mathrm{t} \overline{\mathrm{t}}}$ range, is mildly positive. This allows discriminating both between the signal and background processes and between the $\mathrm{H}$ and 


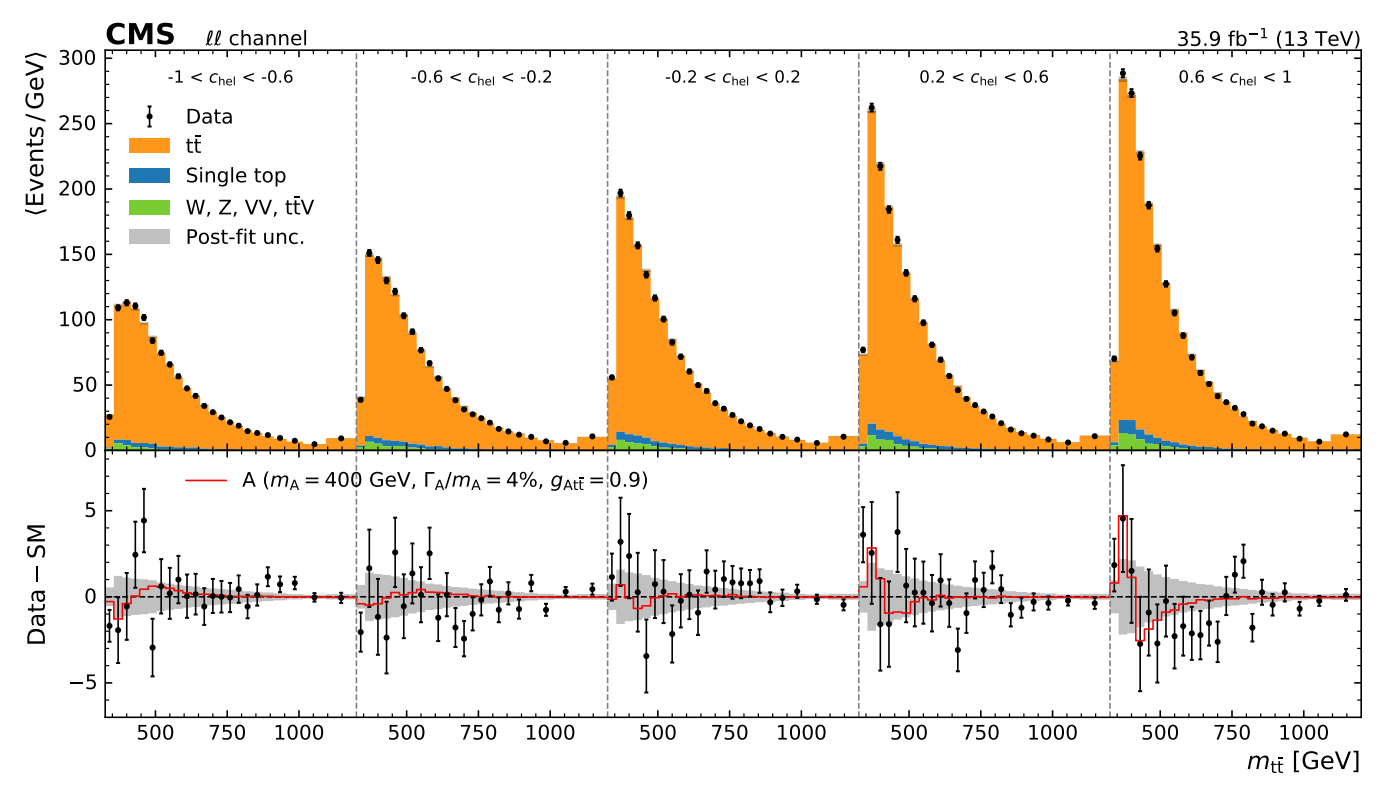

Figure 4. Observed and expected distributions of the observables exploited in the dilepton channel. The expected distributions have been obtained with a background-only fit to the data, and an approximate post-fit uncertainty is shown with a gray band. The impact of the best-fit signal is included in the lower panel for illustration.

A states. The observed and post-fit predicted distributions used in the dilepton channel are shown in figure 4 .

\section{$5 \quad$ Systematic uncertainties}

Various sources of uncertainty affect the distributions of the observables used to search for a heavy Higgs boson signal. Below we describe the experimental and theoretical systematic effects considered in the analysis. In the statistical evaluation discussed in section 6, each source of uncertainty corresponds to a nuisance parameter in a binned maximumlikelihood fit to the distributions of the observables in data. Uncertainties that affect only the normalization are modeled using log-normal constraints, while Gaussian constraints are imposed for nuisance parameters that control all other uncertainties. Unless stated otherwise, all uncertainties are evaluated on signal as well as background processes and treated as fully correlated among the processes and lepton channels. The uncertainties are summarized in table 3 .

The uncertainty due to the jet $p_{\mathrm{T}}$ scale [29] is evaluated by varying the corresponding corrections within their uncertainties. The events are reanalyzed, by reapplying the event selection and recalculating all kinematic quantities. A total of 19 independent jet momentum correction uncertainties affecting jets in the tracker acceptance are considered. As the jet $p_{\mathrm{T}}$ resolution in simulation is smeared to match the resolution observed in data, a corresponding uncertainty is evaluated. An uncertainty in the unclustered component of $p_{\mathrm{T}}^{\text {miss }}$ is computed by shifting the energies of PF candidates not clustered into jets with $p_{\mathrm{T}}>15 \mathrm{GeV}$ according to the energy resolution for each type of PF candidate [34]. Uncer- 
tainties in the b tagging efficiency scale factors applied to simulated events are evaluated by varying them within the respective uncertainties [31]. The scale factors for heavy-flavor (b and c) jets are varied independently of those for light-flavor jets. The uncertainties in the trigger scale factors as well as the electron and muon identification scale factors are considered [32, 33], where the lepton identification also includes effects originating from the isolation requirement and the track reconstruction. The uncertainties in the trigger efficiency scale factors for the single-electron and single-muon channels are considered not correlated with each other, but each of them is independently correlated with the uncertainty in the trigger scale factors in the dilepton channel, with a $50 \%$ correlation coefficient. Effects due to the uncertainty in the distribution of the number of pileup interactions are evaluated by varying the effective inelastic proton-proton cross section in the simulation by $4.6 \%$ from its nominal value. The uncertainty in the integrated luminosity amounts to $2.5 \%[35]$ and affects the normalization of all simulated processes.

The prediction of the SM t $\bar{t}$ production, the main background process in the analysis, is affected by various sources of theoretical uncertainties. The overall normalization of the SM t $t \bar{t}$ background is assigned an uncertainty of $6 \%$, from the NNLO + NNLL QCD cross section calculation $[51,52]$ that is used to normalize the events. The effect of the choice of the renormalization and factorization scales, $\mu_{\mathrm{R}}$ and $\mu_{\mathrm{F}}$, in the matrix element is evaluated by varying these scales independently by a factor of 2 and $1 / 2$. The effect on the acceptance is considered, but the effect on the cross section is ignored as it is already included in the considered uncertainty in the cross section. The renormalization scales used in the parton shower simulation of initial-state radiation (ISR) and final-state radiation (FSR) are also varied independently by a factor of 2 in each direction. The effect of the uncertainty in the amount of ISR, as well as in the underlying event tune used in the simulation, was found not to be statistically significant. The uncertainty in the top quark mass is considered by shifting $m_{\mathrm{t}}$ in the simulation by $\pm 3 \mathrm{GeV}$ and rescaling the induced variations by a factor of $1 / 6$ to emulate a more realistic top quark mass uncertainty of $0.5 \mathrm{GeV}$ [74]. The uncertainty in the matching scale between the matrix element and the parton shower is evaluated by varying the POWHEG parameter, $h_{\text {damp }}$, that controls the suppression of radiation of additional high- $p_{\mathrm{T}}$ jets [50]. The nominal value of $h_{\text {damp }}$ in the simulation is $1.58 \mathrm{~m}_{\mathrm{t}}$, and the varied values are $0.99 \mathrm{~m}_{\mathrm{t}}$ and $2.24 \mathrm{~m}_{\mathrm{t}}$. The uncertainty arising from the choice of the PDF set is evaluated by reweighting the simulated t $\bar{t}$ events using 100 replicas of the NNPDF3.0 set. A principal component analysis is performed on the variations from the PDF replicas to construct two base variations, such that the deviation from the nominal distribution given by each replica can be described as a linear combination of the base variations. The uncertainty in the $\alpha_{S}$ parameter used in the PDF set induces a third independent PDF variation. The uncertainty accounting for the mismodeling of the $p_{\mathrm{T}}$ spectrum of top quarks is evaluated by varying the two parameters used in the top quark $p_{\mathrm{T}}$ reweighting function.

The renormalization and factorization scale uncertainties in the heavy Higgs boson signal simulation are treated independently for the resonant and interference components. Compared to the alternative of varying the scales for the two components simultaneously, we found this to be the more conservative option. The effect on the acceptance as well as on the cross section is considered. Since the simulated samples have been generated at 
LO accuracy, the total effect on the cross section reaches values in excess of $30 \%$. Other theoretical uncertainties in the signal, such as the uncertainties in $m_{\mathrm{t}}$ or PDF, are neglected as they are expected to be small compared to this variation.

The expected yields for most of the non-t $\bar{t}$ background processes are derived using theoretical predictions for the cross sections at NLO or higher accuracy. The uncertainties assumed in the normalization of these processes are conservative and always exceed those of the corresponding theoretical computations. For the single top quark production in the $t$ (tW) channel we assign an uncertainty of $20(15) \%$, which is based on the measurements

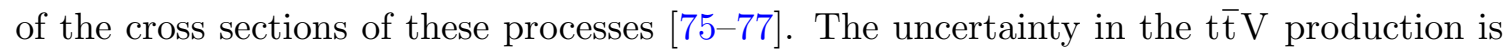
taken to be $30 \%$, which covers the uncertainties of the experimental measurements [78, 79]. To account for the fact that this search probes a restricted region of the phase space of the corresponding processes, we assign uncertainties of $50 \%$ for $\mathrm{W}+$ jets and $\mathrm{Z} / \gamma^{*}+$ jets production (only in the single-lepton channel) and 30\% for the diboson production. Finally, a $20 \%$ uncertainty is used for the $s$-channel single top quark production, for which no measurement at the LHC exists. The adopted conservative normalization uncertainties have little impact on the sensitivity of this search due to the small contribution of these processes.

In cases where the normalization of a background process is estimated using a datadriven method, the corresponding uncertainty is determined by the same method. For the Drell-Yan background in the dilepton channel we assign a $30 \%$ uncertainty, from the variation in the scale factors when derived with and without the requirements on $p_{\mathrm{T}}^{\text {miss }}$, the jet $\mathrm{b}$ tag decisions, and the $\mathrm{t} \overline{\mathrm{t}}$ reconstruction. The normalization of the QCD multijet background, which is only relevant in the single-lepton channel, is assigned an uncertainty of $+100 /-50 \%$, independently in the single-electron and single-muon channels. It covers the statistical uncertainty in the underlying fit and the difference between the data-driven and MC-based estimations.

The nominal background prediction is affected by the limited size of the simulated MC event samples. This statistical uncertainty is evaluated using the "light" BarlowBeeston method [80], by introducing one additional nuisance parameter per bin of the 2D distribution of the observables.

Several systematic variations in the background, most notably those constructed from dedicated MC samples, are affected by statistical fluctuations. We suppress these fluctuations by smoothing the relative deviations from the nominal background distribution of $m_{\mathrm{t} \overline{\mathrm{t}}}$ and the angular variable. The up and down deviations for each independent uncertainty are assumed to be symmetric in shape, but allowed to differ in the overall size. The symmetrized deviation is smoothed using a version of the LOWESS algorithm (LOcally WEighted Scatterplot Smoothing) [81, 82]. In the vicinity of each bin of the 2D distribution, the symmetrized deviation is fitted with a plane using a weighted least squares fit, in which nearby bins receive larger weights. The smoothed deviation obtained in this way is rescaled to account for the overall size of the input up or down deviation, and applied to the nominal background expectation in the given bin. A similar procedure is also applied to all signal distributions. The resulting distributions are then used in the subsequent analysis to evaluate the systematic uncertainty under consideration. 


\begin{tabular}{|c|c|c|c|}
\hline Uncertainty (\# of parameters) & Type & Affected process & Correlation \\
\hline Jet $p_{\mathrm{T}}$ scale $(19)$ & shape & All & All \\
\hline Jet $p_{\mathrm{T}}$ resolution & shape & All & All \\
\hline Unclustered $p_{\mathrm{T}}^{\text {miss }}$ & shape & All & All \\
\hline $\mathrm{b}$ tagging heavy-flavor jets & shape & All & All \\
\hline $\mathrm{b}$ tagging light-flavor jets & shape & All & All \\
\hline Pileup & shape & All & All \\
\hline Electron identification & shape & All & All \\
\hline Muon identification & shape & All & All \\
\hline Single-electron trigger & shape & All & $\mathrm{e}, \ell \ell$ \\
\hline Single-muon trigger & shape & All & $\mu, \ell \ell$ \\
\hline Luminosity calibration & norm. & All & All \\
\hline Renorm. scale SM t $\overline{\mathrm{t}}$ & shape & $\mathrm{SM} t \overline{\mathrm{t}}$ & All \\
\hline Fact. scale SM t $\overline{\mathrm{t}}$ & shape & $\mathrm{SM} t \overline{\mathrm{t}}$ & All \\
\hline Parton shower FSR t $\bar{t}$ & shape & $\mathrm{SM} t \overline{\mathrm{t}}$ & All \\
\hline$h_{\mathrm{damp}}$ & shape & $\mathrm{SM} t \overline{\mathrm{t}}$ & All \\
\hline Top quark mass & shape & $\mathrm{SM} t \overline{\mathrm{t}}$ & All \\
\hline Top quark $p_{\mathrm{T}}(2)$ & shape & $\mathrm{SM} t \overline{\mathrm{t}}$ & All \\
\hline $\mathrm{PDF}(3)$ & shape & $\mathrm{SM} t \overline{\mathrm{t}}$ & All \\
\hline Renorm. scale res. signal & shape & Resonant signal & All \\
\hline Renorm. scale int. signal & shape & Interference signal & All \\
\hline Fact. scale res. signal & shape & Resonant signal & All \\
\hline Fact. scale int. signal & shape & Interference signal & All \\
\hline SM tét norm. & norm. & $\mathrm{SM} t \overline{\mathrm{t}}$ & All \\
\hline Single top $t$ channel norm. & norm. & Single top $t$ channel & $\ell$ \\
\hline Single top $s$ channel norm. & norm. & Single top $s$ channel & $\ell$ \\
\hline Single top tW channel norm. & norm. & Single top tW channel & All \\
\hline $\mathrm{W}+$ jets norm. & norm. & $\mathrm{W}+$ jets & $\ell$ \\
\hline $\mathrm{Z} / \gamma^{*}+$ jets norm. & norm. & $\mathrm{Z} / \gamma^{*}+$ jets & $\ell$ \\
\hline $\mathrm{Z} / \gamma^{*}+$ jets norm. from data & norm. & $\mathrm{Z} / \gamma^{*}+$ jets & $\ell \ell$ \\
\hline Diboson norm. & norm. & Diboson & All \\
\hline $\mathrm{t} \overline{\mathrm{t} V}$ norm. & norm. & $\mathrm{t} \overline{\mathrm{t}} \mathrm{V}$ & All \\
\hline QCD multijet norm. from data, e & norm. & QCD multijet & e \\
\hline QCD multijet norm. from data, $\mu$ & norm. & QCD multijet & $\mu$ \\
\hline MC statistical uncertainty (365) & shape & All & No \\
\hline
\end{tabular}

Table 3. The systematic uncertainties considered in the analysis, indicating the number of corresponding nuisance parameters (when more than one) in the statistical model, the type (affecting shape or only normalization), the affected processes, and the correlation among the lepton channels. Uncertainties tagged in the last column with "All" are correlated among the single-lepton and dilepton channels. In case an uncertainty is only applicable to the single-electron, the single-muon, the single-lepton, or the dilepton channel, they are indicated with e, $\mu, \ell, \ell \ell$, respectively. 
In general, the relative importance of different systematic uncertainties depends greatly on the signal hypothesis, especially the mass of the heavy Higgs boson. Typically, among the uncertainties with the largest impact are the signal theoretical uncertainties and some of the jet momentum correction uncertainties. Close to the t $\bar{t}$ production threshold, the variations in $m_{\mathrm{t}}$ and the $h_{\text {damp }}$ parameter become important, while for larger $m_{\Phi}$ the PDF, $\mu_{\mathrm{R}}$, and $\mu_{\mathrm{F}}$ variations in the SM t $\overline{\mathrm{t}}$ background can have significant impacts. For certain signal hypotheses, the signal distribution partially resembles one of a minor background; in such cases the variation of the normalization of the respective background becomes relevant. In addition, MC statistical uncertainties, when grouped together, often outweigh every other individual uncertainty.

\section{$6 \quad$ Results}

To evaluate the consistency of the observed data with the presence of a signal, we perform a statistical analysis using the $2 \mathrm{D}$ binned distribution of $\left(m_{\mathrm{t} \overline{\mathrm{t}}},\left|\cos \theta_{\mathrm{t}_{\ell}}^{*}\right|\right)$ in the single-electron and the single-muon channels separately, and the $2 \mathrm{D}$ binned distribution of $\left(m_{\mathrm{t} \overline{\mathrm{t}}}, c_{\mathrm{hel}}\right)$ in the combined dilepton channel. The single-lepton and dilepton channels do not overlap as they correspond to orthogonal lepton selection criteria.

The statistical model is defined by the likelihood function

$$
\begin{aligned}
L(\mu, \mathbf{p}, \boldsymbol{\nu}) & =\left(\prod_{i} \frac{\lambda_{i}^{n_{i}}(\mu, \mathbf{p}, \boldsymbol{\nu})}{n_{i} !} \mathrm{e}^{-\lambda_{i}(\mu, \mathbf{p}, \boldsymbol{\nu})}\right) G(\boldsymbol{\nu}), \\
\lambda_{i}(\mu, \mathbf{p}, \boldsymbol{\nu}) & =\mu \sum_{\Phi=\mathrm{H}, \mathrm{A}}\left(g_{\Phi \mathrm{t} \overline{\mathrm{t}}}^{4} s_{R, i}^{\Phi}\left(m_{\Phi}, \Gamma_{\Phi}, \boldsymbol{\nu}\right)+g_{\Phi \mathrm{t} \overline{\mathrm{t}}}^{2} s_{I, i}^{\Phi}\left(m_{\Phi}, \Gamma_{\Phi}, \boldsymbol{\nu}\right)\right)+b_{i}(\boldsymbol{\nu}),
\end{aligned}
$$

with $b_{i}$ denoting the combined background yield in a given bin $i, s_{R, i}^{\Phi}$ and $s_{I, i}^{\Phi}$ the signal yields in a given bin for the resonant and interference part, respectively, $\boldsymbol{\nu}$ the vector of nuisance parameters (on which the signal and background yields generally depend), $n_{i}$ the observed yield, and $g_{\Phi \mathrm{t} \overline{\mathrm{t}}}$ the coupling strength modifiers given by eq. (1.1). The parameters of the signal model (mass $m_{\Phi}$, width $\Gamma_{\Phi}$, and $g_{\Phi t \bar{t}}$ ) are collectively denoted by vector $\mathbf{p}$. Eq. (6.1) is kept generic by including contributions from both CP states. As there is no interference between them, the corresponding signal distributions are trivially added together. We also introduce an auxiliary overall signal strength modifier $\mu$, which rescales the full beyond the SM (BSM) contribution. This allows testing different signal hypotheses in a computationally efficient way, as will be detailed below. The external constraints on the nuisance parameters are taken into account in this likelihood via a product of corresponding probability density functions, $G(\boldsymbol{\nu})$.

The background-only model is constructed by setting $\mu=0$ in eq. (6.1). To quantify the level of agreement between it and observed data, we perform a goodness-of-fit test based on the so-called "saturated model" [83]. This yields a $p$-value of 0.43 , indicating a good overall agreement.

We perform scans over the parameters of the signal models, p. A variant of the LHC profile likelihood ratio test statistic $\tilde{q}_{\mu}$ from refs. $[84,85]$ is utilized:

$$
\tilde{q}_{\mu, \mathbf{p}}=-2 \ln \frac{L\left(\mu, \mathbf{p}, \hat{\boldsymbol{\nu}}_{\mu, \mathbf{p}}\right)}{L\left(\hat{\mu}_{\mathbf{p}}, \mathbf{p}, \hat{\boldsymbol{\nu}}_{\mathbf{p}}\right)}, \quad 0 \leqslant \hat{\mu}_{\mathbf{p}} \leqslant \mu .
$$


The test statistic is expressed in terms of the auxiliary parameter $\mu$ in eq. (6.1), in which the statistical model is linear, while the parameters $\mathbf{p}$ are kept fixed at their values being probed in the scan. The likelihood in the numerator is maximized with respect to the nuisance parameters, and $\hat{\boldsymbol{\nu}}_{\mu, \mathbf{p}}$ denotes the vector of their values at the maximum. A similar notation is used in the denominator, where the likelihood is maximized with respect to both $\mu$ and $\boldsymbol{\nu}$, under the additional constraint $0 \leqslant \hat{\mu}_{\mathbf{p}} \leqslant \mu$. The requirement $\hat{\mu}_{\mathbf{p}} \geqslant 0$ excludes from the consideration cases in which the shape of the overall BSM contribution gets flipped, resulting in a qualitatively different effect from what is targeted in this search. The condition $\hat{\mu}_{\mathbf{p}} \leqslant \mu$ prevents the exclusion of a signal hypothesis if the data are more compatible with a model that predicts the BSM contribution of a similar shape but a larger overall size.

For each signal hypothesis $\mathbf{p}$, we perform a test according to the $\mathrm{CL}_{\mathrm{s}}$ criterion $[86,87]$. This is done for $\mu=1$ in eq. (6.2), which reproduces the nominal signal expectation. We profit from the known asymptotic approximation [84] for distributions of the adopted test statistic to construct these distributions in a computationally efficient way. If the $\mathrm{CL}_{\mathrm{s}}$ value computed for $\mu=1$ and given $\mathbf{p}$ is found to be smaller than 0.05 , the point $\mathbf{p}$ is said to be excluded at $95 \%$ confidence level (CL).

\subsection{Interpolation and extrapolation of signal masses and widths}

To construct expected signal distributions for every point encountered in the scans, we apply an interpolation in mass and width of the heavy Higgs boson, starting from the reference generated points. This is done independently for the $m_{\mathrm{t} \overline{\mathrm{t}}}$ distribution in each bin of the angular variable. We consider the resonant part of the signal and the interference separately and further split the interference contribution in two according to the sign of the per-event generator weight. A change in the mass results in a horizontal shift of the $m_{\mathrm{t} \overline{\mathrm{t}}}$ distribution. The interpolation in this observable is implemented with a nonlinear morphing algorithm [88]. On the other hand, the effect of a change in $\Gamma_{\Phi}$ is evaluated with an independent interpolation in each bin.

The signal model parameter scan may reach values of $\Gamma_{\Phi} / m_{\Phi}$ below $2.5 \%$, the lowest value considered in the simulated signal samples. Since the reconstructed $m_{\mathrm{t} \overline{\mathrm{t}}}$ resolution is about $17 \%$ or worse, the shape of the $m_{\mathrm{t} \overline{\mathrm{t}}}$ distribution for a signal with such low widths does not differ from the one corresponding to $\Gamma_{\Phi} / m_{\Phi}=2.5 \%$. Hence, for scan points with $\Gamma_{\Phi} / m_{\Phi}<2.5 \%$ it is sufficient to use the distributions for $\Gamma_{\Phi} / m_{\Phi}=2.5 \%$ and only scale the cross sections appropriately.

\subsection{Model-independent interpretation}

Constraints on the coupling strength modifier $g_{\Phi \mathrm{t} \overline{\mathrm{t}}}$ are derived as a function of the mass and width of the heavy Higgs boson, for each CP state independently. The coupling modifier for the other CP state in eq. (6.1) is set to zero to exclude it from the statistical model. The scan is performed for $m_{\Phi}$ between 400 and $750 \mathrm{GeV}$ and $\Gamma_{\Phi} / m_{\Phi}$ between 0.5 and $25 \%$. The mass and width interpolation described in section 6.1 is performed in scan points other than those corresponding to the generated signal samples. Coupling strength values up to 3 are probed to guarantee that the amplitudes preserve perturbative unitarity for all 
calculations, in accordance with the lower bound $\tan \beta=1 / g_{\mathrm{At} \overline{\mathrm{t}}} \gtrsim 0.3$ given in ref. [4] in the context of $2 \mathrm{HDM}$.

The constraints obtained on $g_{\Phi \mathrm{t} \bar{t}}$ are presented in figures 5 and 6 for the scalar and the pseudoscalar scenarios, respectively. Since the total width $\Gamma_{\Phi}$ is kept fixed during the scans and the partial width of $\Phi \rightarrow \mathrm{t} \overline{\mathrm{t}}$ is proportional to $g_{\Phi \mathrm{t} \overline{\mathrm{t}}}^{2}$, in some regions the partial width can exceed $\Gamma_{\Phi}$. These unphysical regions are marked in the figures with hatched lines. In some cases the observed exclusion for a given mass does not extend continuously all the way to the largest probed $g_{\Phi \mathrm{t} \overline{\mathrm{t}}}=3$ (e.g., $m_{\mathrm{H}} \approx 700 \mathrm{GeV}$ in the panel for $\Gamma_{\mathrm{H}} / m_{\mathrm{H}}=25 \%$ in figure 5). This is due to the strong dependence of the shape of the signal distribution on the value of the coupling strength modifier. For some values of $g_{\Phi t \bar{t}}$ the shape becomes compatible with systematic variations in the background.

As evident from figure 6 , there is a signal-like excess for the pseudoscalar hypotheses with low masses. The largest deviation from the SM background is observed for a pseudoscalar Higgs boson with a mass of $400 \mathrm{GeV}$ and a total relative width of $4 \%$, with a local significance of $3.5 \pm 0.3$ standard deviations. Figure 7 shows scans of $-2 \ln \left[L\left(g_{\mathrm{At} \overline{\mathrm{t}}}\right) / L_{\mathrm{SM}}\right]$ for this hypothesis, as a function of the coupling modifier $g_{\mathrm{At} \overline{\mathrm{t}}}$. The likelihoods $L\left(g_{\mathrm{At} \overline{\mathrm{t}}}\right)$ and $L_{\mathrm{SM}}$ are given by eq. (6.1). They are computed for $\mu=1$ and 0 respectively and in both cases maximized with respect to all nuisance parameters. The scans are shown for the observed data, as well as for the expectations under the background-only hypothesis and in the presence of the signal. In the latter case the coupling modifier is set to the value obtained in the combined fit, $g_{\mathrm{At} \overline{\mathrm{t}}} \approx 0.9$. In this case and in general, the expected sensitivity is comparable between the single-lepton and dilepton channels. The single-lepton channel is slightly more sensitive for the scalar hypotheses and in the case of the pseudoscalar hypotheses with larger masses. However, figure 7 demonstrates that the observed excess is driven by the dilepton channel, which is also supported by the comparisons between the observed and expected distributions in figures 3 and 4 .

When accounting for the look-elsewhere effect [89] in the mass, total width, and CP state of the heavy Higgs boson, the significance of the excess is 1.9 standard deviations, which corresponds to a $p$-value of 0.028 . We note that higher-order electroweak corrections

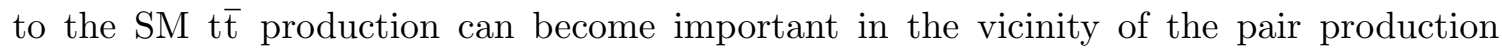
threshold [90] and may account for the excess.

\subsection{Interpretation in the hMSSM}

For the hMSSM, we perform a scan over the two model parameters, $m_{\mathrm{A}}$ and $\tan \beta$. Both $\mathrm{A}$ and $\mathrm{H}$ bosons are included. For each point, the coupling strength modifiers $g_{\mathrm{At \overline {t }}}$ and $g_{\mathrm{Ht \overline {t }}}$, the mass $m_{\mathrm{H}}$, and the widths of the two heavy Higgs bosons are determined with the 2HDMC program. The CP-even state is more massive of the two, but the mass separation $\Delta m=m_{\mathrm{H}}-m_{\mathrm{A}}$ decreases with $m_{\mathrm{A}}$ and $\tan \beta$. Typical values of $\Delta m / m_{\mathrm{A}}$ vary from $\approx 20 \%$ for $m_{\mathrm{A}}=400 \mathrm{GeV}, \tan \beta=0.5$ to $\approx 1 \%$ for $m_{\mathrm{A}}=700 \mathrm{GeV}, \tan \beta=2$. The scan is performed for $m_{\mathrm{A}}$ between 400 and $700 \mathrm{GeV}$ in steps of $12.5 \mathrm{GeV}$, and $\tan \beta$ between 0.4 and 5.0 in steps of 0.2 . Similarly to what was done above, the lower boundary $\tan \beta>0.4$ is imposed to assure perturbative unitarity [4]. The mass and width interpolation described 

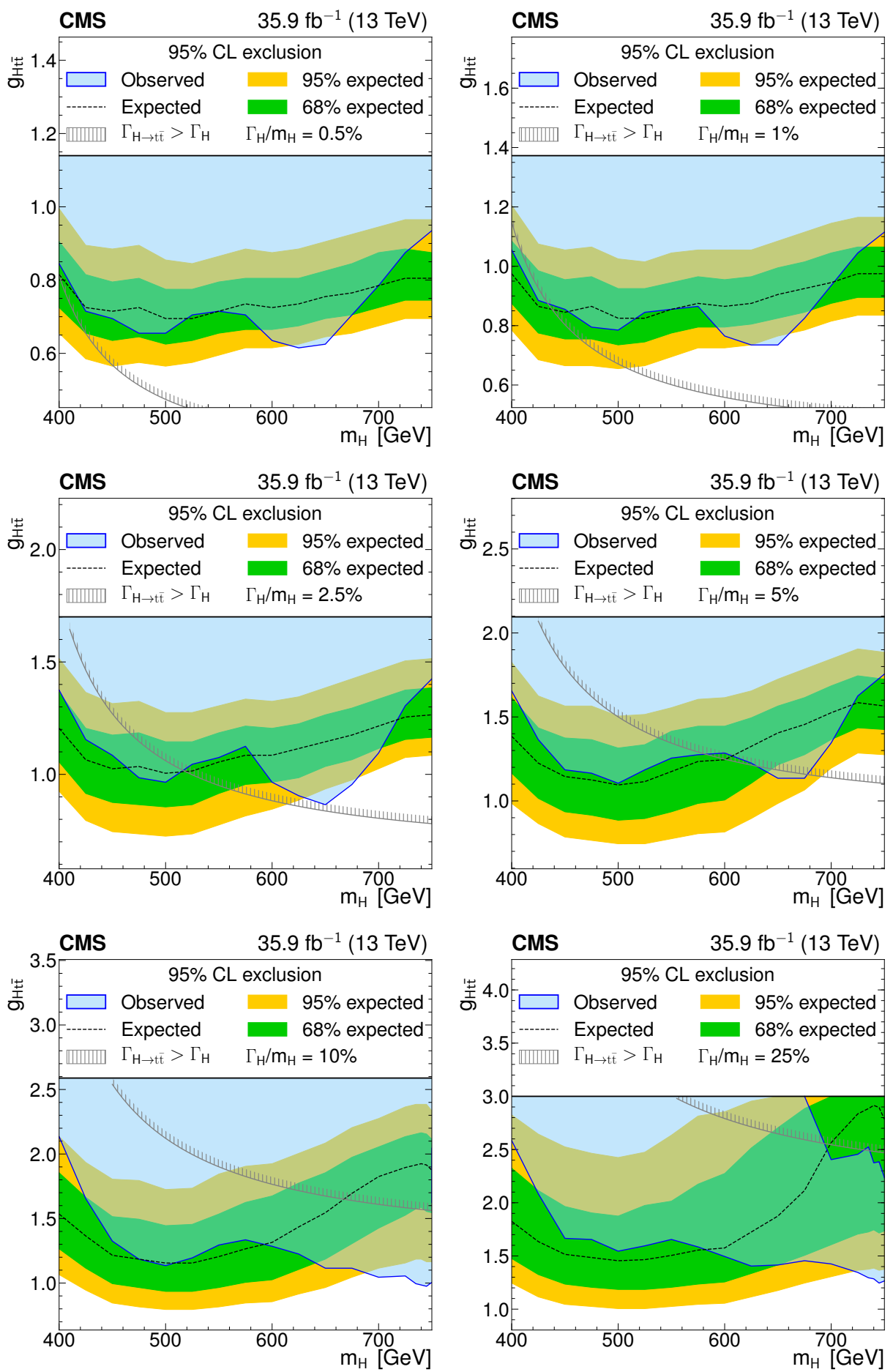

Figure 5. Model-independent constraints on the coupling strength modifier as a function of the heavy scalar boson mass, for relative widths of $0.5,1,2.5,5,10$, and $25 \%$. The observed constraints are indicated by the blue shaded area. The inner (green) band and the outer (yellow) band indicate the regions containing 68 and 95\%, respectively, of the distribution of constraints expected under the background-only hypothesis. The unphysical region of phase space in which the partial width $\Gamma_{\mathrm{H} \rightarrow \mathrm{t} \overline{\mathrm{t}}}$ becomes larger than the total width is indicated by the hatched lines. 

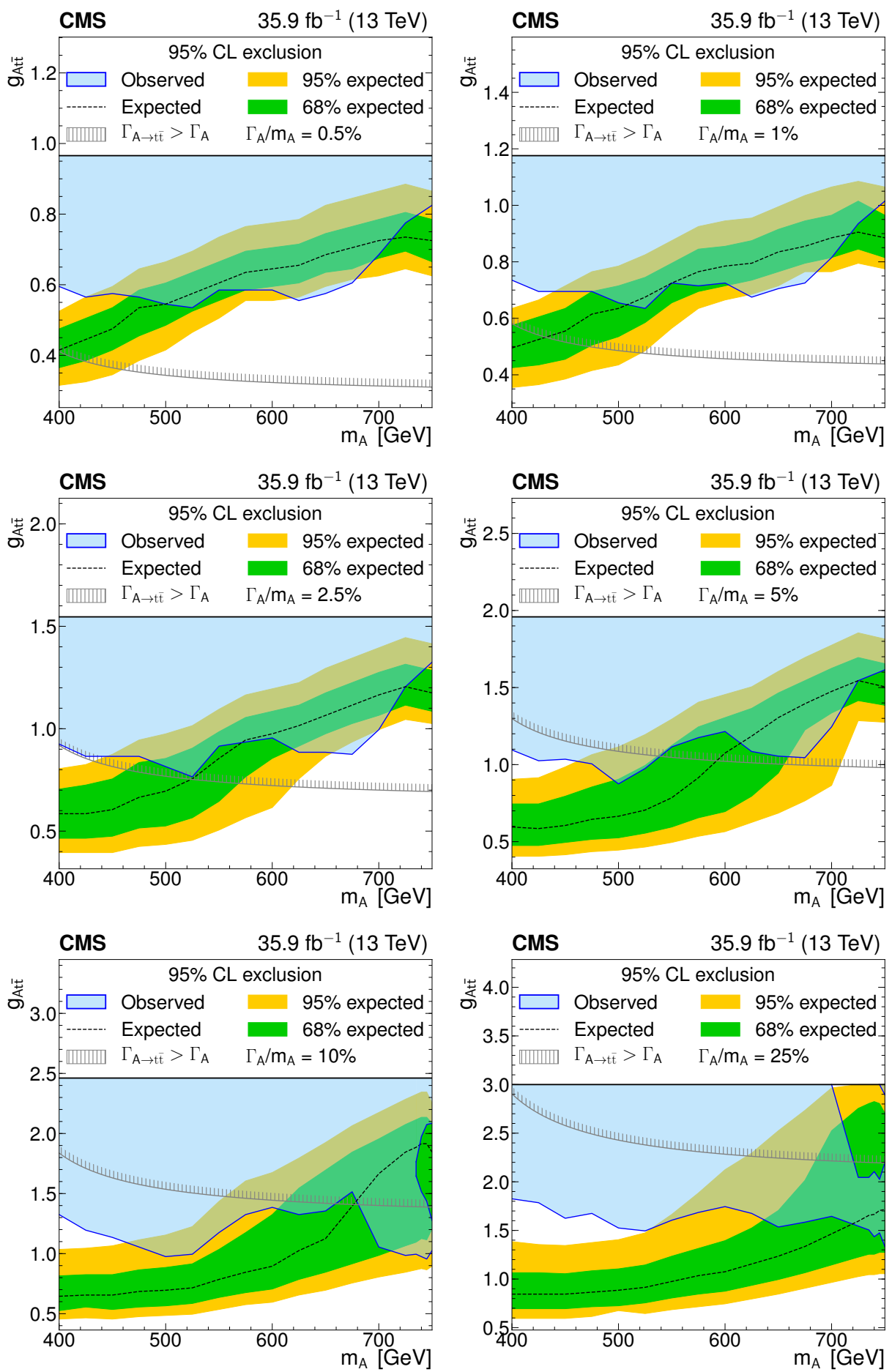

Figure 6. Model-independent constraints on the coupling strength modifier as a function of the heavy pseudoscalar boson mass, for relative widths of $0.5,1,2.5,5,10$, and $25 \%$. The observed constraints are indicated by the blue shaded area. The inner (green) band and the outer (yellow) band indicate the regions containing 68 and $95 \%$, respectively, of the distribution of constraints expected under the background-only hypothesis. The unphysical region of phase space in which the partial width $\Gamma_{\mathrm{A} \rightarrow \mathrm{t} \overline{\mathrm{t}}}$ becomes larger than the total width is indicated by the hatched lines. 


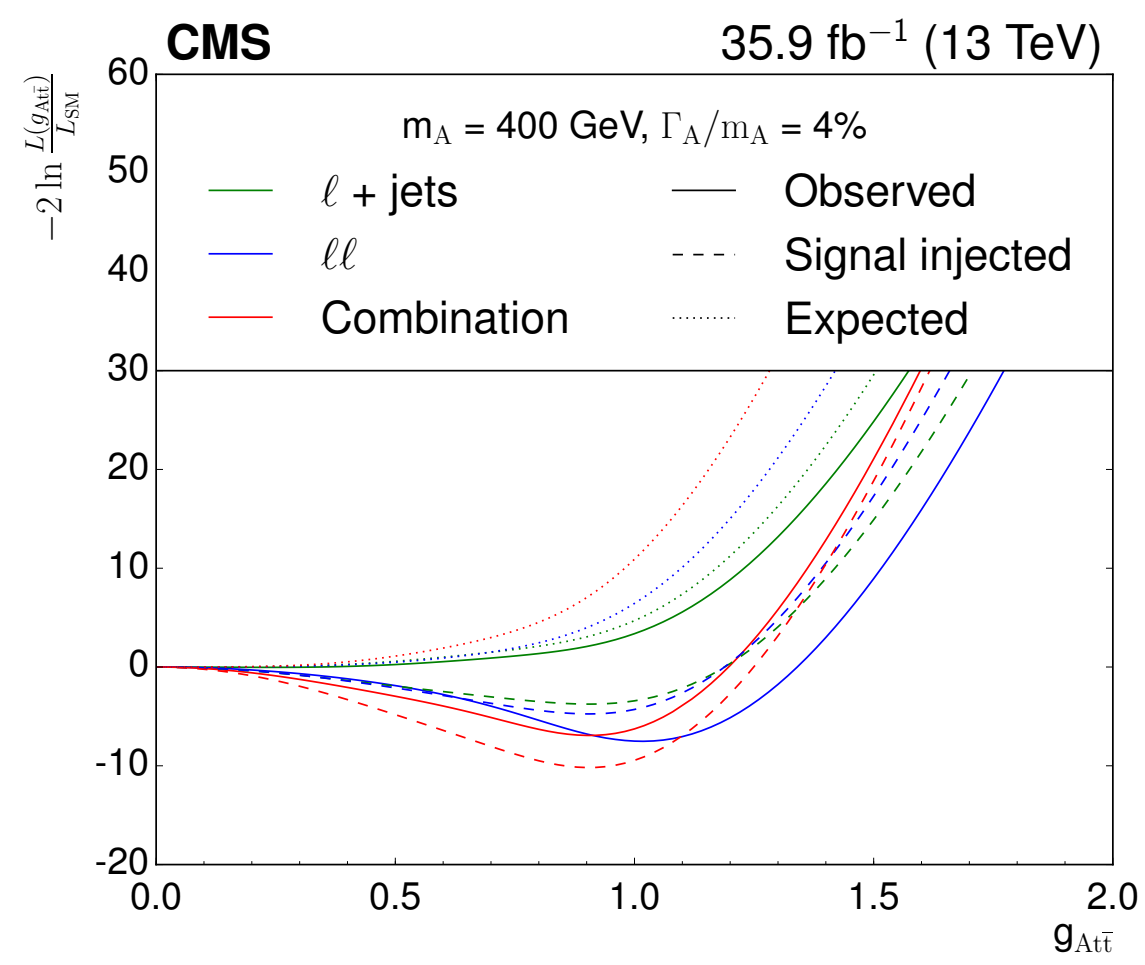

Figure 7. Scans of profiled likelihood for the pseudoscalar hypothesis with $m_{\mathrm{A}}=400 \mathrm{GeV}$ and $\Gamma_{\mathrm{A}} / m_{\mathrm{A}}=4 \%$. The scans are shown for the single- and dilepton channels separately, as well as for the combination.

in section 6.1 is performed in scan points other than those corresponding to the generated signal samples.

The expected and observed exclusions in the $\left(m_{\mathrm{A}}, \tan \beta\right)$ plane are presented in figure 8. The upper boundary of the observed (expected) exclusion in $\tan \beta$ varies from 1.0 (2.3) at $m_{\mathrm{A}}=400 \mathrm{GeV}$ to $1.5(0.8)$ at $m_{\mathrm{A}}=700 \mathrm{GeV}$. The tension between the observed exclusion and the expectation at low $m_{\mathrm{A}}$ is a manifestation of the excess discussed above. These results can be compared to those of the search for $\mathrm{H}^{ \pm} \rightarrow \mathrm{t} \overline{\mathrm{b}} / \overline{\mathrm{t}} \mathrm{b}$ in ref. [23], which were also interpreted in the hMSSM benchmark, setting constraints in the $\left(m_{\mathrm{H}^{ \pm}}, \tan \beta\right)$ plane. Translating the results from ref. [23] in terms of $m_{\mathrm{A}}$, the present analysis observes a more stringent exclusion in $\tan \beta$ for $m_{\mathrm{A}} \approx 700 \mathrm{GeV}$, while the exclusion for $m_{\mathrm{A}} \approx 400 \mathrm{GeV}$ is substantially weaker than in the reference due to the observed signal-like deviation. The expected exclusion is tighter than in ref. [23] throughout the considered $m_{\mathrm{A}}$ range.

\section{Summary}

Results are presented for the search for additional heavy Higgs bosons decaying to a pair of top quarks. A data sample recorded with the CMS detector at $\sqrt{s}=13 \mathrm{TeV}$, corresponding to an integrated luminosity of $35.9 \mathrm{fb}^{-1}$, is analyzed. The final states with one or two leptons are utilized. The invariant mass of the reconstructed $t \bar{t}$ system as well as angular variables sensitive to the spin of the new boson are used to search for the signal, while taking into account the interference with the standard model $t \bar{t}$ production. 


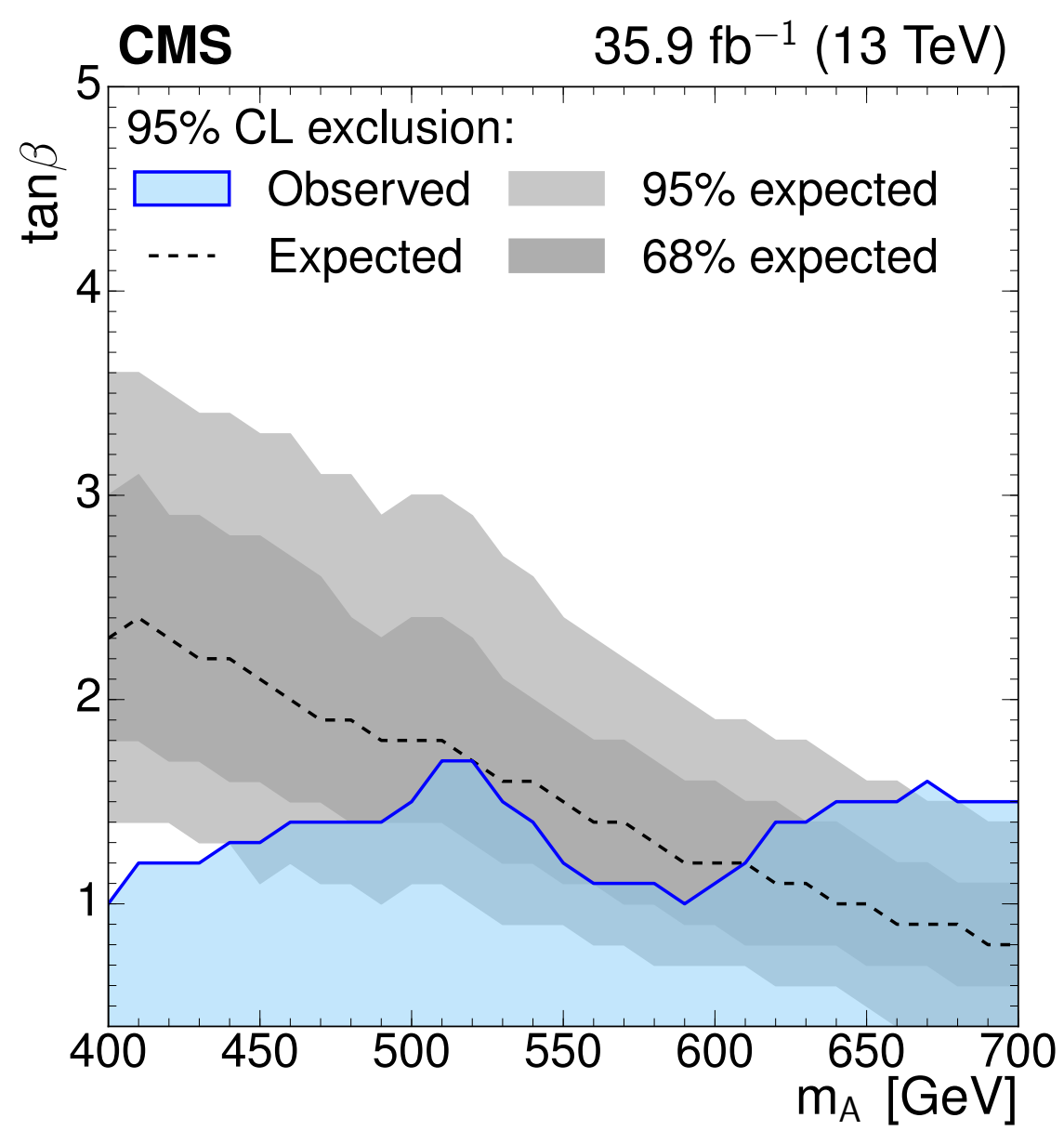

Figure 8. Exclusion in the $\left(m_{\mathrm{A}}, \tan \beta\right)$ plane of the hMSSM. The inner (dark gray) band and the outer (light gray) band indicate the regions containing 68 and 95\%, respectively, of the distribution of constraints expected under the background-only hypothesis. The observed excluded region is indicated by the blue shaded area. Both $\mathrm{H}$ and $\mathrm{A}$ boson signals are included with masses and widths that correspond to a given point in the plane.

A moderate signal-like deviation is observed for the hypothesis of a pseudoscalar Higgs boson with the mass $m_{\mathrm{A}} \approx 400 \mathrm{GeV}$. After accounting for the look-elsewhere effect, its significance is 1.9 standard deviations. Further improvements of the theoretical description of the standard model t $\overline{\mathrm{t}}$ process in the vicinity of the production threshold will be needed to clarify the origin of this deviation.

Constraints on the strength of the coupling of the sought-for boson to top quarks are reported, separately for the scalar and pseudoscalar cases, for the mass ranging from 400 to $750 \mathrm{GeV}$ and the total relative width from 0.5 to $25 \%$. These are the most stringent constraints on this coupling to date. The results are also interpreted in the hMSSM scenario in the minimal supersymmetric standard model. This search probes the values of $m_{\mathrm{A}}$ from 400 to $700 \mathrm{GeV}$ and excludes, at $95 \%$ confidence level, the region with values of $\tan \beta$ below 1.0 to 1.5 , depending on $m_{\mathrm{A}}$. This extends the exclusion obtained in previous searches. 


\section{Acknowledgments}

We congratulate our colleagues in the CERN accelerator departments for the excellent performance of the LHC and thank the technical and administrative staffs at CERN and at other CMS institutes for their contributions to the success of the CMS effort. In addition, we gratefully acknowledge the computing centers and personnel of the Worldwide LHC Computing Grid for delivering so effectively the computing infrastructure essential to our analyses. Finally, we acknowledge the enduring support for the construction and operation of the LHC and the CMS detector provided by the following funding agencies: BMBWF and FWF (Austria); FNRS and FWO (Belgium); CNPq, CAPES, FAPERJ, FAPERGS, and FAPESP (Brazil); MES (Bulgaria); CERN; CAS, MoST, and NSFC (China); COLCIENCIAS (Colombia); MSES and CSF (Croatia); RPF (Cyprus); SENESCYT (Ecuador); MoER, ERC IUT, PUT and ERDF (Estonia); Academy of Finland, MEC, and HIP (Finland); CEA and CNRS/IN2P3 (France); BMBF, DFG, and HGF (Germany); GSRT (Greece); NKFIA (Hungary); DAE and DST (India); IPM (Iran); SFI (Ireland); INFN (Italy); MSIP and NRF (Republic of Korea); MES (Latvia); LAS (Lithuania); MOE and UM (Malaysia); BUAP, CINVESTAV, CONACYT, LNS, SEP, and UASLP-FAI (Mexico); MOS (Montenegro); MBIE (New Zealand); PAEC (Pakistan); MSHE and NSC (Poland); FCT (Portugal); JINR (Dubna); MON, RosAtom, RAS, RFBR, and NRC KI (Russia); MESTD (Serbia); SEIDI, CPAN, PCTI, and FEDER (Spain); MOSTR (Sri Lanka); Swiss Funding Agencies (Switzerland); MST (Taipei); ThEPCenter, IPST, STAR, and NSTDA (Thailand); TUBITAK and TAEK (Turkey); NASU and SFFR (Ukraine); STFC (United Kingdom); DOE and NSF (U.S.A.).

Individuals have received support from the Marie-Curie program and the European Research Council and Horizon 2020 Grant, contract Nos. 675440, 752730, and 765710 (European Union); the Leventis Foundation; the A.P. Sloan Foundation; the Alexander von Humboldt Foundation; the Belgian Federal Science Policy Office; the Fonds pour la Formation à la Recherche dans l'Industrie et dans l'Agriculture (FRIA-Belgium); the Agentschap voor Innovatie door Wetenschap en Technologie (IWT-Belgium); the F.R.S.-FNRS and FWO (Belgium) under the "Excellence of Science - EOS" — be.h project n. 30820817; the Beijing Municipal Science \& Technology Commission, No. Z181100004218003; the Ministry of Education, Youth and Sports (MEYS) of the Czech Republic; the Lendület ("Momentum") Program and the János Bolyai Research Scholarship of the Hungarian Academy of Sciences, the New National Excellence Program ÚNKP, the NKFIA research grants 123842, 123959, 124845, 124850, 125105, 128713, 128786, and 129058 (Hungary); the Council of Science and Industrial Research, India; the HOMING PLUS program of the Foundation for Polish Science, cofinanced from European Union, Regional Development Fund, the Mobility Plus program of the Ministry of Science and Higher Education, the National Science Center (Poland), contracts Harmonia 2014/14/M/ST2/00428, Opus 2014/13/B/ST2/02543, 2014/15/B/ST2/03998, and 2015/19/B/ST2/02861, Sonatabis 2012/07/E/ST2/01406; the National Priorities Research Program by Qatar National Research Fund; the Ministry of Science and Education, grant no. 3.2989.2017 (Russia); the Programa Estatal de Fomento de la Investigación Científica y Técnica de Excelencia María 
de Maeztu, grant MDM-2015-0509 and the Programa Severo Ochoa del Principado de Asturias; the Thalis and Aristeia programs cofinanced by EU-ESF and the Greek NSRF; the Rachadapisek Sompot Fund for Postdoctoral Fellowship, Chulalongkorn University and the Chulalongkorn Academic into Its 2nd Century Project Advancement Project (Thailand); the Welch Foundation, contract C-1845; and the Weston Havens Foundation (U.S.A.).

Open Access. This article is distributed under the terms of the Creative Commons Attribution License (CC-BY 4.0), which permits any use, distribution and reproduction in any medium, provided the original author(s) and source are credited.

\section{References}

[1] ATLAS collaboration, Observation of a new particle in the search for the Standard Model Higgs boson with the ATLAS detector at the LHC, Phys. Lett. B 716 (2012) 1 [arXiv: 1207.7214] [INSPIRE].

[2] CMS collaboration, Observation of a new boson at a mass of $125 \mathrm{GeV}$ with the CMS experiment at the LHC, Phys. Lett. B 716 (2012) 30 [arXiv:1207.7235] [INSPIRE].

[3] CMS collaboration, Observation of a new boson with mass near $125 \mathrm{GeV}$ in pp collisions at $\sqrt{s}=7$ and $8 \mathrm{TeV}$, JHEP 06 (2013) 081 [arXiv: 1303.4571] [INSPIRE].

[4] G.C. Branco et al., Theory and phenomenology of two-Higgs-doublet models, Phys. Rept. 516 (2012) 1 [arXiv: 1106.0034] [INSPIRE].

[5] J. Wess and B. Zumino, Supergauge transformations in four-dimensions, Nucl. Phys. B 70 (1974) 39 [INSPIRE].

[6] S. Dimopoulos and H. Georgi, Softly broken supersymmetry and SU(5), Nucl. Phys. B 193 (1981) 150 [INSPIRE].

[7] K. Huitu et al., Probing pseudo-Goldstone dark matter at the LHC, Phys. Rev. D 100 (2019) 015009 [arXiv: 1812.05952] [INSPIRE].

[8] M. Mühlleitner, M.O.P. Sampaio, R. Santos and J. Wittbrodt, Phenomenological comparison of models with extended Higgs sectors, JHEP 08 (2017) 132 [arXiv:1703.07750] [INSPIRE].

[9] J. Abdallah et al., Simplified models for dark matter searches at the LHC, Phys. Dark Univ. 9-10 (2015) 8 [arXiv:1506.03116] [INSPIRE].

[10] C. Arina et al., A comprehensive approach to dark matter studies: exploration of simplified top-philic models, JHEP 11 (2016) 111 [arXiv:1605.09242] [INSPIRE].

[11] N. Craig, J. Galloway and S. Thomas, Searching for signs of the second Higgs doublet, arXiv:1305.2424 [INSPIRE].

[12] K. Lane and W. Shepherd, Natural stabilization of the Higgs boson's mass and alignment, Phys. Rev. D 99 (2019) 055015 [arXiv: 1808.07927] [inSPIRE].

[13] A. Djouadi et al., The post-Higgs MSSM scenario: Habemus MSSM?, Eur. Phys. J. C 73 (2013) 2650 [arXiv: 1307.5205] [INSPIRE].

[14] K.J.F. Gaemers and F. Hoogeveen, Higgs production and decay into heavy flavors with the gluon fusion mechanism, Phys. Lett. B 146 (1984) 347 [INSPIRE].

[15] D. Dicus, A. Stange and S. Willenbrock, Higgs decay to top quarks at hadron colliders, Phys. Lett. B 333 (1994) 126 [hep-ph/9404359] [INSPIRE]. 
[16] W. Bernreuther, M. Flesch and P. Haberl, Signatures of Higgs bosons in the top quark decay channel at hadron colliders, Phys. Rev. D 58 (1998) 114031 [hep-ph/9709284] [INSPIRE].

[17] M. Carena and Z. Liu, Challenges and opportunities for heavy scalar searches in the t $\bar{t}$ channel at the LHC, JHEP 11 (2016) 159 [arXiv: 1608.07282] [INSPIRE].

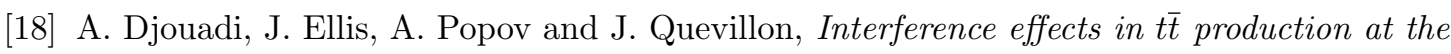
LHC as a window on new physics, JHEP 03 (2019) 119 [arXiv:1901.03417] [INSPIRE].

[19] ATLAS collaboration, Search for heavy Higgs bosons $A / H$ decaying to a top quark pair in pp collisions at $\sqrt{s}=8 \mathrm{TeV}$ with the ATLAS detector, Phys. Rev. Lett. 119 (2017) 191803 [arXiv: 1707.06025] [INSPIRE].

[20] CMS collaboration, Search for physics beyond the standard model in events with two leptons of same sign, missing transverse momentum and jets in proton-proton collisions at $\sqrt{s}=13 \mathrm{TeV}$, Eur. Phys. J. C 77 (2017) 578 [arXiv: 1704.07323] [INSPIRE].

[21] ATLAS collaboration, Search for heavy particles decaying into top-quark pairs using lepton-plus-jets events in proton-proton collisions at $\sqrt{s}=13 \mathrm{TeV}$ with the ATLAS detector, Eur. Phys. J. C 78 (2018) 565 [arXiv: 1804.10823] [INSPIRE].

[22] CMS collaboration, Search for $t \bar{t}$ resonances in highly boosted lepton+jets and fully hadronic final states in proton-proton collisions at $\sqrt{s}=13$ TeV, JHEP 07 (2017) 001 [arXiv: 1704.03366] [INSPIRE].

[23] ATLAS collaboration, Search for charged Higgs bosons decaying into top and bottom quarks at $\sqrt{s}=13 \mathrm{TeV}$ with the ATLAS detector, JHEP 11 (2018) 085 [arXiv:1808.03599] [INSPIRE].

[24] CMS collaboration, Search for a charged Higgs boson in pp collisions at $\sqrt{s}=8 \mathrm{TeV}$, JHEP 11 (2015) 018 [arXiv: 1508.07774] [INSPIRE].

[25] CMS collaboration, The CMS experiment at the CERN LHC, 2008 JINST 3 S08004 [INSPIRE].

[26] M. Cacciari, G.P. Salam and G. Soyez, The anti- $k_{\mathrm{T}}$ jet clustering algorithm, JHEP 04 (2008) 063 [arXiv:0802.1189] [INSPIRE].

[27] M. Cacciari, G.P. Salam and G. Soyez, FastJet user manual, Eur. Phys. J. C 72 (2012) 1896 [arXiv: 1111.6097] [INSPIRE].

[28] CMS collaboration, Particle-flow reconstruction and global event description with the CMS detector, 2017 JINST 12 P10003 [arXiv:1706.04965] [INSPIRE].

[29] CMS collaboration, Jet energy scale and resolution in the CMS experiment in pp collisions at $8 \mathrm{TeV}, 2017$ JINST $12 \mathrm{P} 02014$ [arXiv:1607.03663] [INSPIRE].

[30] CMS collaboration, Jet algorithms performance in $13 \mathrm{TeV}$ data, CMS-PAS-JME-16-003 (2016).

[31] CMS collaboration, Identification of heavy-flavour jets with the CMS detector in pp collisions at $13 \mathrm{TeV}, 2018$ JINST $13 \mathrm{P} 05011$ [arXiv:1712.07158] [INSPIRE].

[32] CMS collaboration, Performance of electron reconstruction and selection with the CMS detector in proton-proton collisions at $\sqrt{s}=8 \mathrm{TeV}, 2015$ JINST $10 \mathrm{P} 06005$ [arXiv: 1502.02701] [INSPIRE].

[33] CMS collaboration, Performance of the CMS muon detector and muon reconstruction with proton-proton collisions at $\sqrt{s}=13 \mathrm{TeV}, 2018$ JINST 13 P06015 [arXiv:1804.04528] [INSPIRE]. 
[34] CMS collaboration, Performance of missing transverse momentum reconstruction in proton-proton collisions at $\sqrt{s}=13$ TeV using the CMS detector, 2019 JINST 14 P07004 [arXiv: 1903.06078] [INSPIRE].

[35] CMS collaboration, CMS luminosity measurements for the 2016 data taking period, CMS-PAS-LUM-17-001 (2017).

[36] CMS collaboration, The CMS trigger system, 2017 JINST 12 P01020 [arXiv:1609.02366] [INSPIRE].

[37] J. Alwall et al., The automated computation of tree-level and next-to-leading order differential cross sections and their matching to parton shower simulations, JHEP 07 (2014) 079 [arXiv: 1405.0301] [INSPIRE].

[38] M. Spira, A. Djouadi, D. Graudenz and P.M. Zerwas, Higgs boson production at the LHC, Nucl. Phys. B 453 (1995) 17 [hep-ph/9504378] [INSPIRE].

[39] NNPDF collaboration, Parton distributions for the LHC Run II, JHEP 04 (2015) 040 [arXiv: 1410.8849] [INSPIRE].

[40] T. Sjöstrand et al., An introduction to PYTHIA 8.2, Comput. Phys. Commun. 191 (2015) 159 [arXiv: 1410.3012] [INSPIRE].

[41] P. Skands, S. Carrazza and J. Rojo, Tuning PYTHIA 8.1: the Monash 2013 tune, Eur. Phys. J. C 74 (2014) 3024 [arXiv:1404.5630] [INSPIRE].

[42] CMS collaboration, Event generator tunes obtained from underlying event and multiparton scattering measurements, Eur. Phys. J. C 76 (2016) 155 [arXiv:1512.00815] [InSPIRE].

[43] B. Hespel, F. Maltoni and E. Vryonidou, Signal background interference effects in heavy scalar production and decay to a top-anti-top pair, JHEP 10 (2016) 016 [arXiv:1606.04149] [INSPIRE].

[44] R.V. Harlander, S. Liebler and H. Mantler, SusHi: a program for the calculation of Higgs production in gluon fusion and bottom-quark annihilation in the Standard Model and the MSSM, Comput. Phys. Commun. 184 (2013) 1605 [arXiv:1212.3249] [INSPIRE].

[45] D. Eriksson, J. Rathsman and O. Stal, 2HDMC: two-Higgs-doublet model calculator physics and manual, Comput. Phys. Commun. 181 (2010) 189 [arXiv:0902.0851] [INSPIRE].

[46] P. Nason, A new method for combining NLO QCD with shower Monte Carlo algorithms, JHEP 11 (2004) 040 [hep-ph/0409146] [INSPIRE].

[47] S. Frixione, P. Nason and C. Oleari, Matching NLO QCD computations with parton shower simulations: the POWHEG method, JHEP 11 (2007) 070 [arXiv:0709.2092] [INSPIRE].

[48] S. Alioli, P. Nason, C. Oleari and E. Re, A general framework for implementing NLO calculations in shower Monte Carlo programs: the POWHEG BOX, JHEP 06 (2010) 043 [arXiv: 1002.2581] [INSPIRE].

[49] J.M. Campbell, R.K. Ellis, P. Nason and E. Re, Top-pair production and decay at NLO matched with parton showers, JHEP 04 (2015) 114 [arXiv: 1412.1828] [INSPIRE].

[50] CMS collaboration, Investigations of the impact of the parton shower tuning in PYTHIA 8 in the modelling of $t \bar{t}$ at $\sqrt{s}=8$ and $13 \mathrm{TeV}$, CMS-PAS-TOP-16-021 (2016).

[51] M. Czakon and A. Mitov, Top++: a program for the calculation of the top-pair cross-section at hadron colliders, Comput. Phys. Commun. 185 (2014) 2930 [arXiv:1112.5675] [INSPIRE]. 
[52] M. Botje et al., The PDF4LHC working group interim recommendations, arXiv:1101.0538 [INSPIRE].

[53] A.D. Martin, W.J. Stirling, R.S. Thorne and G. Watt, Uncertainties on $\alpha_{s}$ in global PDF analyses and implications for predicted hadronic cross sections, Eur. Phys. J. C 64 (2009) 653 [arXiv:0905.3531] [INSPIRE].

[54] J. Gao et al., CT10 next-to-next-to-leading order global analysis of QCD, Phys. Rev. D 89 (2014) 033009 [arXiv: 1302.6246] [INSPIRE].

[55] NNPDF collaboration, Parton distributions with LHC data, Nucl. Phys. B 867 (2013) 244 [arXiv: 1207.1303] [INSPIRE].

[56] CMS collaboration, Measurement of differential cross sections for top quark pair production using the lepton+jets final state in proton-proton collisions at $13 \mathrm{TeV}$, Phys. Rev. D 95 (2017) 092001 [arXiv: 1610.04191] [INSPIRE].

[57] CMS collaboration, Measurement of normalized differential $t \bar{t}$ cross sections in the dilepton channel from pp collisions at $\sqrt{s}=13 \mathrm{TeV}$, JHEP 04 (2018) 060 [arXiv:1708.07638] [INSPIRE].

[58] M. Aliev et al., HATHOR: HAdronic Top and Heavy quarks crOss section calculatoR, Comput. Phys. Commun. 182 (2011) 1034 [arXiv:1007.1327] [INSPIRE].

[59] P. Kant et al., HatHor for single top-quark production: updated predictions and uncertainty estimates for single top-quark production in hadronic collisions, Comput. Phys. Commun. 191 (2015) 74 [arXiv:1406.4403] [INSPIRE].

[60] N. Kidonakis, Top quark production, in the proceedings of the Helmholtz International Summer School on Physics of Heavy Quarks and Hadrons (HQ2013), July 15-28, Dubna, Russia Federation (2014), arXiv:1311.0283.

[61] J. Alwall et al., Comparative study of various algorithms for the merging of parton showers and matrix elements in hadronic collisions, Eur. Phys. J. C 53 (2008) 473 [arXiv:0706.2569] [INSPIRE].

[62] K. Melnikov and F. Petriello, Electroweak gauge boson production at hadron colliders through $O\left(\alpha_{s}^{2}\right)$, Phys. Rev. D 74 (2006) 114017 [hep-ph/0609070] [INSPIRE].

[63] Y. Li and F. Petriello, Combining QCD and electroweak corrections to dilepton production in FEWZ, Phys. Rev. D 86 (2012) 094034 [arXiv: 1208.5967] [InSPIRE].

[64] S. Frixione and B.R. Webber, Matching NLO QCD computations and parton shower simulations, JHEP 06 (2002) 029 [hep-ph/0204244] [INSPIRE].

[65] T. Gehrmann et al., $W^{+} W^{-}$production at hadron colliders in next to next to leading order QCD, Phys. Rev. Lett. 113 (2014) 212001 [arXiv: 1408.5243] [INSPIRE].

[66] J.M. Campbell and R.K. Ellis, MCFM for the Tevatron and the LHC, Nucl. Phys. Proc. Suppl. 205-206 (2010) 10 [arXiv:1007.3492] [INSPIRE].

[67] GEANT4 collaboration, GEANT4 - a simulation toolkit, Nucl. Instrum. Meth. A 506 (2003) 250 [INSPIRE].

[68] B.A. Betchart, R. Demina and A. Harel, Analytic solutions for neutrino momenta in decay of top quarks, Nucl. Instrum. Meth. A 736 (2014) 169 [arXiv:1305.1878] [InSPIRE].

[69] A. Loginov, Strategies of data-driven estimations of $t \bar{t}$ backgrounds in ATLAS, Nuovo Cim. C 033 (2010) 175 [INSPIRE]. 
[70] CMS collaboration, Measurements of t $\bar{t}$ differential cross sections in proton-proton collisions at $\sqrt{s}=13$ TeV using events containing two leptons, JHEP 02 (2019) 149 [arXiv: 1811.06625] [INSPIRE].

[71] CMS collaboration, Measurement of the $t \bar{t}$ production cross section and the top quark mass in the dilepton channel in pp collisions at $\sqrt{s}=7$ TeV, JHEP 07 (2011) 049 [arXiv:1105.5661] [INSPIRE].

[72] CMS collaboration, Measurement of the differential cross section for top quark pair production in pp collisions at $\sqrt{s}=8 \mathrm{TeV}$, Eur. Phys. J. C 75 (2015) 542 [arXiv: 1505.04480] [INSPIRE].

[73] W. Bernreuther, A. Brandenburg, Z.G. Si and P. Uwer, Top quark pair production and decay at hadron colliders, Nucl. Phys. B 690 (2004) 81 [hep-ph/0403035] [InSPIRE].

[74] CMS collaboration, Measurement of the top quark mass using proton-proton data at $\sqrt{s}=7$ and 8 TeV, Phys. Rev. D 93 (2016) 072004 [arXiv: 1509.04044] [InSPIRE].

[75] ATLAS collaboration, Measurement of the inclusive cross-sections of single top-quark and top-antiquark $t$-channel production in pp collisions at $\sqrt{s}=13$ TeV with the ATLAS detector, JHEP 04 (2017) 086 [arXiv: 1609.03920] [INSPIRE].

[76] CMS collaboration, Measurement of the single top quark and antiquark production cross sections in the $t$ channel and their ratio in proton-proton collisions at $\sqrt{s}=13$ TeV, Phys. Lett. B 800 (2020) 135042 [arXiv:1812.10514] [INSPIRE].

[77] CMS collaboration, Measurement of the production cross section for single top quarks in association with $W$ bosons in proton-proton collisions at $\sqrt{s}=13 \mathrm{TeV}$, JHEP 10 (2018) 117 [arXiv: 1805.07399] [INSPIRE].

[78] CMS collaboration, Measurement of the cross section for top quark pair production in association with a $W$ or $Z$ boson in proton-proton collisions at $\sqrt{s}=13$ TeV, JHEP 08 (2018) 011 [arXiv: 1711.02547] [INSPIRE].

[79] ATLAS collaboration, Measurement of the $t \bar{t} Z$ and $t \bar{t} W$ cross sections in proton-proton collisions at $\sqrt{s}=13$ TeV with the ATLAS detector, Phys. Rev. D 99 (2019) 072009 [arXiv: 1901.03584] [INSPIRE].

[80] R.J. Barlow and C. Beeston, Fitting using finite Monte Carlo samples, Comput. Phys. Commun. 77 (1993) 219 [INSPIRE].

[81] W.S. Cleveland, Robust locally weighted regression and smoothing scatterplots, J. Am. Stat. Assoc. 74 (1979) 829.

[82] W.S. Cleveland and S.J. Devlin, Locally-weighted regression: an approach to regression analysis by local fitting, J. Am. Stat. Assoc. 83 (1988) 596.

[83] S. Baker and R.D. Cousins, Clarification of the use of $\chi^{2}$ and likelihood functions in fits to histograms, Nucl. Instrum. Meth. 221 (1984) 437 [INSPIRE].

[84] G. Cowan, K. Cranmer, E. Gross and O. Vitells, Asymptotic formulae for likelihood-based tests of new physics, Eur. Phys. J. C 71 (2011) 1554 [Erratum ibid. C 73 (2013) 2501] [arXiv:1007.1727] [INSPIRE].

[85] ATLAS, CMS collaborations and LHC Higgs Combination Group, Procedure for the LHC Higgs boson search combination in Summer 2011, CMS-NOTE-2011-005 (2011).

[86] T. Junk, Confidence level computation for combining searches with small statistics, Nucl. Instrum. Meth. A 434 (1999) 435 [hep-ex/9902006] [InSPIRE]. 
[87] A.L. Read, Presentation of search results: the $C L_{s}$ technique, J. Phys. G 28 (2002) 2693 [INSPIRE].

[88] M. Baak, S. Gadatsch, R. Harrington and W. Verkerke, Interpolation between multi-dimensional histograms using a new non-linear moment morphing method, Nucl. Instrum. Meth. A 771 (2015) 39 [arXiv:1410.7388] [INSPIRE].

[89] L. Demortier, $P$ values and nuisance parameters, in the proceedings of Statistical issues for LHC physics (PHYSTAT-LHC), June 27-29, Geneva, Switzerland (2007).

[90] J.H. Kühn, A. Scharf and P. Uwer, Weak interactions in top-quark pair production at hadron colliders: an update, Phys. Rev. D 91 (2015) 014020 [arXiv:1305.5773] [INSPIRE]. 


\section{The CMS collaboration}

\section{Yerevan Physics Institute, Yerevan, Armenia}

A.M. Sirunyan ${ }^{\dagger}$, A. Tumasyan

\section{Institut für Hochenergiephysik, Wien, Austria}

W. Adam, F. Ambrogi, T. Bergauer, J. Brandstetter, M. Dragicevic, J. Erö, A. Escalante Del Valle, M. Flechl, R. Frühwirth ${ }^{1}$, M. Jeitler ${ }^{1}$, N. Krammer, I. Krätschmer, D. Liko, T. Madlener, I. Mikulec, N. Rad, J. Schieck ${ }^{1}$, R. Schöfbeck, M. Spanring, D. Spitzbart, W. Waltenberger, C.-E. Wulz ${ }^{1}$, M. Zarucki

Institute for Nuclear Problems, Minsk, Belarus

V. Drugakov, V. Mossolov, J. Suarez Gonzalez

\section{Universiteit Antwerpen, Antwerpen, Belgium}

M.R. Darwish, E.A. De Wolf, D. Di Croce, X. Janssen, J. Lauwers, A. Lelek, M. Pieters, H. Rejeb Sfar, H. Van Haevermaet, P. Van Mechelen, S. Van Putte, N. Van Remortel

\section{Vrije Universiteit Brussel, Brussel, Belgium}

F. Blekman, E.S. Bols, S.S. Chhibra, J. D'Hondt, J. De Clercq, D. Lontkovskyi, S. Lowette, I. Marchesini, S. Moortgat, L. Moreels, Q. Python, K. Skovpen, S. Tavernier, W. Van Doninck, P. Van Mulders, I. Van Parijs

\section{Université Libre de Bruxelles, Bruxelles, Belgium}

D. Beghin, B. Bilin, H. Brun, B. Clerbaux, G. De Lentdecker, H. Delannoy, B. Dorney,

L. Favart, A. Grebenyuk, A.K. Kalsi, J. Luetic, A. Popov, N. Postiau, E. Starling, L. Thomas, C. Vander Velde, P. Vanlaer, D. Vannerom

\section{Ghent University, Ghent, Belgium}

T. Cornelis, D. Dobur, I. Khvastunov' ${ }^{2}$, M. Niedziela, C. Roskas, D. Trocino, M. Tytgat, W. Verbeke, B. Vermassen, M. Vit, N. Zaganidis

Université Catholique de Louvain, Louvain-la-Neuve, Belgium

O. Bondu, G. Bruno, C. Caputo, P. David, C. Delaere, M. Delcourt, A. Giammanco, V. Lemaitre, A. Magitteri, J. Prisciandaro, A. Saggio, M. Vidal Marono, P. Vischia, J. Zobec

\section{Centro Brasileiro de Pesquisas Fisicas, Rio de Janeiro, Brazil}

F.L. Alves, G.A. Alves, G. Correia Silva, C. Hensel, A. Moraes, P. Rebello Teles

Universidade do Estado do Rio de Janeiro, Rio de Janeiro, Brazil

E. Belchior Batista Das Chagas, W. Carvalho, J. Chinellato ${ }^{3}$, E. Coelho, E.M. Da Costa, G.G. Da Silveira ${ }^{4}$, D. De Jesus Damiao, C. De Oliveira Martins, S. Fonseca De Souza, L.M. Huertas Guativa, H. Malbouisson, J. Martins ${ }^{5}$, D. Matos Figueiredo, M. Medina Jaime ${ }^{6}$, M. Melo De Almeida, C. Mora Herrera, L. Mundim, H. Nogima, W.L. Prado Da Silva, L.J. Sanchez Rosas, A. Santoro, A. Sznajder, M. Thiel, E.J. Tonelli Manganote ${ }^{3}$, F. Torres Da Silva De Araujo, A. Vilela Pereira 
Universidade Estadual Paulista ${ }^{a}$, Universidade Federal do $\mathrm{ABC}^{b}$, São Paulo, Brazil

S. Ahuja ${ }^{a}$, C.A. Bernardes ${ }^{a}$, L. Calligaris ${ }^{a}$, T.R. Fernandez Perez Tomei ${ }^{a}$, E.M. Gregores ${ }^{b}{ }$ D.S. Lemos, P.G. Mercadante ${ }^{b}$, S.F. Novaes ${ }^{a}$, SandraS. Padula ${ }^{a}$

Institute for Nuclear Research and Nuclear Energy, Bulgarian Academy of Sciences, Sofia, Bulgaria

A. Aleksandrov, G. Antchev, R. Hadjiiska, P. Iaydjiev, A. Marinov, M. Misheva, M. Rodozov, M. Shopova, G. Sultanov

University of Sofia, Sofia, Bulgaria

M. Bonchev, A. Dimitrov, T. Ivanov, L. Litov, B. Pavlov, P. Petkov

Beihang University, Beijing, China

W. Fang ${ }^{7}$, X. Gao ${ }^{7}$, L. Yuan

Department of Physics, Tsinghua University, Beijing, China

Z. Hu, Y. Wang

Institute of High Energy Physics, Beijing, China

M. Ahmad, G.M. Chen, H.S. Chen, M. Chen, C.H. Jiang, D. Leggat, H. Liao, Z. Liu, S.M. Shaheen ${ }^{8}$, A. Spiezia, J. Tao, E. Yazgan, H. Zhang, S. Zhang ${ }^{8}$, J. Zhao

State Key Laboratory of Nuclear Physics and Technology, Peking University, Beijing, China

A. Agapitos, Y. Ban, G. Chen, A. Levin, J. Li, L. Li, Q. Li, Y. Mao, S.J. Qian, D. Wang, Q. Wang

Universidad de Los Andes, Bogota, Colombia

C. Avila, A. Cabrera, L.F. Chaparro Sierra, C. Florez, C.F. González Hernández, M.A. Segura Delgado

Universidad de Antioquia, Medellin, Colombia

J. Mejia Guisao, J.D. Ruiz Alvarez, C.A. Salazar González, N. Vanegas Arbelaez

University of Split, Faculty of Electrical Engineering, Mechanical Engineering and Naval Architecture, Split, Croatia

D. Giljanović, N. Godinovic, D. Lelas, I. Puljak, T. Sculac

University of Split, Faculty of Science, Split, Croatia

Z. Antunovic, M. Kovac

Institute Rudjer Boskovic, Zagreb, Croatia

V. Brigljevic, S. Ceci, D. Ferencek, K. Kadija, B. Mesic, M. Roguljic, A. Starodumov ${ }^{9}$, T. Susa

University of Cyprus, Nicosia, Cyprus

M.W. Ather, A. Attikis, E. Erodotou, A. Ioannou, M. Kolosova, S. Konstantinou, G. Mavromanolakis, J. Mousa, C. Nicolaou, F. Ptochos, P.A. Razis, H. Rykaczewski, D. Tsiakkouri 
Charles University, Prague, Czech Republic

M. Finger ${ }^{10}$, M. Finger Jr. ${ }^{10}$, A. Kveton, J. Tomsa

Escuela Politecnica Nacional, Quito, Ecuador

E. Ayala

Universidad San Francisco de Quito, Quito, Ecuador

E. Carrera Jarrin

Academy of Scientific Research and Technology of the Arab Republic of Egypt, Egyptian Network of High Energy Physics, Cairo, Egypt

H. Abdalla ${ }^{11}$, A. Mohamed ${ }^{12}$

National Institute of Chemical Physics and Biophysics, Tallinn, Estonia

S. Bhowmik, A. Carvalho Antunes De Oliveira, R.K. Dewanjee, K. Ehataht, M. Kadastik, M. Raidal, C. Veelken

Department of Physics, University of Helsinki, Helsinki, Finland

P. Eerola, L. Forthomme, H. Kirschenmann, K. Osterberg, M. Voutilainen

Helsinki Institute of Physics, Helsinki, Finland

F. Garcia, J. Havukainen, J.K. Heikkilä, T. Järvinen, V. Karimäki, R. Kinnunen,

T. Lampén, K. Lassila-Perini, S. Laurila, S. Lehti, T. Lindén, P. Luukka, T. Mäenpää,

H. Siikonen, E. Tuominen, J. Tuominiemi

Lappeenranta University of Technology, Lappeenranta, Finland

T. Tuuva

IRFU, CEA, Université Paris-Saclay, Gif-sur-Yvette, France

M. Besancon, F. Couderc, M. Dejardin, D. Denegri, B. Fabbro, J.L. Faure, F. Ferri, S. Ganjour, A. Givernaud, P. Gras, G. Hamel de Monchenault, P. Jarry, C. Leloup, E. Locci, J. Malcles, J. Rander, A. Rosowsky, M.Ö. Sahin, A. Savoy-Navarro ${ }^{13}$, M. Titov

Laboratoire Leprince-Ringuet, CNRS/IN2P3, Ecole Polytechnique, Institut Polytechnique de Paris

C. Amendola, F. Beaudette, P. Busson, C. Charlot, B. Diab, G. Falmagne, R. Granier de Cassagnac, I. Kucher, A. Lobanov, C. Martin Perez, M. Nguyen, C. Ochando, P. Paganini, J. Rembser, R. Salerno, J.B. Sauvan, Y. Sirois, A. Zabi, A. Zghiche

Université de Strasbourg, CNRS, IPHC UMR 7178, Strasbourg, France

J.-L. Agram ${ }^{14}$, J. Andrea, D. Bloch, G. Bourgatte, J.-M. Brom, E.C. Chabert, C. Collard, E. Conte ${ }^{14}$, J.-C. Fontaine ${ }^{14}$, D. Gelé, U. Goerlach, M. Jansová, A.-C. Le Bihan, N. Tonon, P. Van Hove

Centre de Calcul de l'Institut National de Physique Nucleaire et de Physique des Particules, CNRS/IN2P3, Villeurbanne, France

S. Gadrat 
Université de Lyon, Université Claude Bernard Lyon 1, CNRS-IN2P3, Institut de Physique Nucléaire de Lyon, Villeurbanne, France

S. Beauceron, C. Bernet, G. Boudoul, C. Camen, N. Chanon, R. Chierici, D. Contardo, P. Depasse, H. El Mamouni, J. Fay, S. Gascon, M. Gouzevitch, B. Ille, Sa. Jain, F. Lagarde, I.B. Laktineh, H. Lattaud, M. Lethuillier, L. Mirabito, S. Perries, V. Sordini, G. Touquet, M. Vander Donckt, S. Viret

Georgian Technical University, Tbilisi, Georgia

A. Khvedelidze ${ }^{10}$

Tbilisi State University, Tbilisi, Georgia

Z. Tsamalaidze ${ }^{10}$

RWTH Aachen University, I. Physikalisches Institut, Aachen, Germany

C. Autermann, L. Feld, M.K. Kiesel, K. Klein, M. Lipinski, D. Meuser, A. Pauls, M. Preuten, M.P. Rauch, C. Schomakers, J. Schulz, M. Teroerde, B. Wittmer

RWTH Aachen University, III. Physikalisches Institut A, Aachen, Germany

A. Albert, M. Erdmann, S. Erdweg, T. Esch, B. Fischer, R. Fischer, S. Ghosh, T. Hebbeker, K. Hoepfner, H. Keller, L. Mastrolorenzo, M. Merschmeyer, A. Meyer, P. Millet, G. Mocellin, S. Mondal, S. Mukherjee, D. Noll, A. Novak, T. Pook, A. Pozdnyakov, T. Quast, M. Radziej, Y. Rath, H. Reithler, M. Rieger, J. Roemer, A. Schmidt, S.C. Schuler, A. Sharma, S. Thüer, S. Wiedenbeck

RWTH Aachen University, III. Physikalisches Institut B, Aachen, Germany G. Flügge, W. Haj Ahmad ${ }^{15}$, O. Hlushchenko, T. Kress, T. Müller, A. Nehrkorn, A. Nowack, C. Pistone, O. Pooth, D. Roy, H. Sert, A. Stahl ${ }^{16}$

Deutsches Elektronen-Synchrotron, Hamburg, Germany

M. Aldaya Martin, P. Asmuss, I. Babounikau, H. Bakhshiansohi, K. Beernaert, O. Behnke, U. Behrens, A. Bermúdez Martínez, D. Bertsche, A.A. Bin Anuar, K. Borras ${ }^{17}$, V. Botta, A. Campbell, A. Cardini, P. Connor, S. Consuegra Rodríguez, C. Contreras-Campana, V. Danilov, A. De Wit, M.M. Defranchis, C. Diez Pardos, D. Domínguez Damiani, G. Eckerlin, D. Eckstein, T. Eichhorn, A. Elwood, E. Eren, E. Gallo ${ }^{18}$, A. Geiser, J.M. Grados Luyando, A. Grohsjean, M. Guthoff, M. Haranko, A. Harb, A. Jafari, N.Z. Jomhari, H. Jung, A. Kasem ${ }^{17}$, M. Kasemann, H. Kaveh, J. Keaveney, C. Kleinwort, J. Knolle, D. Krücker, W. Lange, T. Lenz, J. Leonard, J. Lidrych, K. Lipka, W. Lohmann ${ }^{19}$, R. Mankel, I.-A. Melzer-Pellmann, A.B. Meyer, M. Meyer, M. Missiroli, G. Mittag, J. Mnich, A. Mussgiller, V. Myronenko, D. Pérez Adán, S.K. Pflitsch, D. Pitzl, A. Raspereza, A. Saibel, M. Savitskyi, V. Scheurer, P. Schütze, C. Schwanenberger, R. Shevchenko, A. Singh, H. Tholen, O. Turkot, A. Vagnerini, M. Van De Klundert, G.P. Van Onsem, R. Walsh, Y. Wen, K. Wichmann, C. Wissing, O. Zenaiev, R. Zlebcik

University of Hamburg, Hamburg, Germany

R. Aggleton, S. Bein, L. Benato, A. Benecke, V. Blobel, T. Dreyer, A. Ebrahimi, A. Fröhlich, C. Garbers, E. Garutti, D. Gonzalez, P. Gunnellini, J. Haller, A. Hinzmann, A. Karavdina, G. Kasieczka, R. Klanner, R. Kogler, N. Kovalchuk, S. Kurz, V. Kutzner, 
J. Lange, T. Lange, A. Malara, D. Marconi, J. Multhaup, C.E.N. Niemeyer, D. Nowatschin, A. Perieanu, A. Reimers, O. Rieger, C. Scharf, P. Schleper, S. Schumann, J. Schwandt, J. Sonneveld, H. Stadie, G. Steinbrück, F.M. Stober, M. Stöver, B. Vormwald, I. Zoi

Karlsruher Institut fuer Technologie, Karlsruhe, Germany

M. Akbiyik, C. Barth, M. Baselga, S. Baur, T. Berger, E. Butz, R. Caspart, T. Chwalek, W. De Boer, A. Dierlamm, K. El Morabit, N. Faltermann, M. Giffels, P. Goldenzweig, A. Gottmann, M.A. Harrendorf, F. Hartmann ${ }^{16}$, U. Husemann, S. Kudella, S. Mitra, M.U. Mozer, Th. Müller, M. Musich, A. Nürnberg, G. Quast, K. Rabbertz, M. Schröder, I. Shvetsov, H.J. Simonis, R. Ulrich, M. Weber, C. Wöhrmann, R. Wolf

Institute of Nuclear and Particle Physics (INPP), NCSR Demokritos, Aghia Paraskevi, Greece

G. Anagnostou, P. Asenov, G. Daskalakis, T. Geralis, A. Kyriakis, D. Loukas, G. Paspalaki National and Kapodistrian University of Athens, Athens, Greece

M. Diamantopoulou, G. Karathanasis, P. Kontaxakis, A. Panagiotou, I. Papavergou, N. Saoulidou, A. Stakia, K. Theofilatos, K. Vellidis

National Technical University of Athens, Athens, Greece

G. Bakas, K. Kousouris, I. Papakrivopoulos, G. Tsipolitis

University of Ioánnina, Ioánnina, Greece

I. Evangelou, C. Foudas, P. Gianneios, P. Katsoulis, P. Kokkas, S. Mallios, K. Manitara, N. Manthos, I. Papadopoulos, J. Strologas, F.A. Triantis, D. Tsitsonis

MTA-ELTE Lendület CMS Particle and Nuclear Physics Group, Eötvös Loránd University, Budapest, Hungary

M. Bartók ${ }^{20}$, M. Csanad, P. Major, K. Mandal, A. Mehta, M.I. Nagy, G. Pasztor, O. Surányi, G.I. Veres

Wigner Research Centre for Physics, Budapest, Hungary

G. Bencze, C. Hajdu, D. Horvath ${ }^{21}$, F. Sikler, T.Á. Vámi, V. Veszpremi, G. Vesztergombi ${ }^{\dagger}$

Institute of Nuclear Research ATOMKI, Debrecen, Hungary

N. Beni, S. Czellar, J. Karancsi ${ }^{20}$, A. Makovec, J. Molnar, Z. Szillasi

Institute of Physics, University of Debrecen, Debrecen, Hungary

P. Raics, D. Teyssier, Z.L. Trocsanyi, B. Ujvari

Eszterhazy Karoly University, Karoly Robert Campus, Gyongyos, Hungary

T. Csorgo, W.J. Metzger, F. Nemes, T. Novak

Indian Institute of Science (IISc), Bangalore, India

S. Choudhury, J.R. Komaragiri, P.C. Tiwari

National Institute of Science Education and Research, HBNI, Bhubaneswar, India

S. Bahinipati ${ }^{23}$, C. Kar, G. Kole, P. Mal, V.K. Muraleedharan Nair Bindhu, A. Nayak ${ }^{24}$, D.K. Sahoo ${ }^{23}$, S.K. Swain 
Panjab University, Chandigarh, India

S. Bansal, S.B. Beri, V. Bhatnagar, S. Chauhan, R. Chawla, N. Dhingra, R. Gupta,

A. Kaur, M. Kaur, S. Kaur, P. Kumari, M. Lohan, M. Meena, K. Sandeep, S. Sharma, J.B. Singh, A.K. Virdi, G. Walia

University of Delhi, Delhi, India

A. Bhardwaj, B.C. Choudhary, R.B. Garg, M. Gola, S. Keshri, Ashok Kumar, S. Malhotra, M. Naimuddin, P. Priyanka, K. Ranjan, Aashaq Shah, R. Sharma

Saha Institute of Nuclear Physics, HBNI, Kolkata, India

R. Bhardwaj ${ }^{25}$, M. Bharti ${ }^{25}$, R. Bhattacharya, S. Bhattacharya, U. Bhawandeep ${ }^{25}$, D. Bhowmik, S. Dey, S. Dutta, S. Ghosh, M. Maity ${ }^{26}$, K. Mondal, S. Nandan, A. Purohit, P.K. Rout, G. Saha, S. Sarkar, T. Sarkar ${ }^{26}$, M. Sharan, B. Singh ${ }^{25}$, S. Thakur ${ }^{25}$

Indian Institute of Technology Madras, Madras, India

P.K. Behera, P. Kalbhor, A. Muhammad, P.R. Pujahari, A. Sharma, A.K. Sikdar

Bhabha Atomic Research Centre, Mumbai, India

R. Chudasama, D. Dutta, V. Jha, V. Kumar, D.K. Mishra, P.K. Netrakanti, L.M. Pant, P. Shukla

Tata Institute of Fundamental Research-A, Mumbai, India

T. Aziz, M.A. Bhat, S. Dugad, G.B. Mohanty, N. Sur, RavindraKumar Verma

Tata Institute of Fundamental Research-B, Mumbai, India

S. Banerjee, S. Bhattacharya, S. Chatterjee, P. Das, M. Guchait, S. Karmakar, S. Kumar,

G. Majumder, K. Mazumdar, N. Sahoo, S. Sawant

Indian Institute of Science Education and Research (IISER), Pune, India

S. Chauhan, S. Dube, V. Hegde, A. Kapoor, K. Kothekar, S. Pandey, A. Rane, A. Rastogi,

S. Sharma

Institute for Research in Fundamental Sciences (IPM), Tehran, Iran

S. Chenarani ${ }^{27}$, E. Eskandari Tadavani, S.M. Etesami ${ }^{27}$, M. Khakzad, M. Mohammadi Najafabadi, M. Naseri, F. Rezaei Hosseinabadi

University College Dublin, Dublin, Ireland

M. Felcini, M. Grunewald

INFN Sezione di Bari ${ }^{a}$, Università di Bari ${ }^{b}$, Politecnico di Bari ${ }^{c}$, Bari, Italy M. Abbrescia ${ }^{a, b}$, R. Aly ${ }^{a, b, 28}$, C. Calabria ${ }^{a, b}$, A. Colaleo ${ }^{a}$, D. Creanza ${ }^{a, c}$, L. Cristella $^{a, b}$, N. De Filippis ${ }^{a, c}$, M. De Palma ${ }^{a, b}$, A. Di Florio ${ }^{a, b}$, L. Fiore $^{a}$, A. Gelmi $^{a, b}$, G. Iaselli $^{a, c}$, M. Ince ${ }^{a, b}$, S. Lezki ${ }^{a, b}$, G. Maggi ${ }^{a, c}$, M. Maggi ${ }^{a}$, G. Miniello ${ }^{a, b}$, S. My ${ }^{a, b}$, S. Nuzzo $^{a, b}$, A. Pompili ${ }^{a, b}$, G. Pugliese ${ }^{a, c}$, R. Radogna ${ }^{a}$, A. Ranieri ${ }^{a}$, G. Selvaggi ${ }^{a, b}$, L. Silvestris ${ }^{a}$, R. Venditti ${ }^{a}$, P. Verwilligen ${ }^{a}$

INFN Sezione di Bologna ${ }^{a}$, Università di Bologna ${ }^{b}$, Bologna, Italy

G. Abbiendi ${ }^{a}$, C. Battilana ${ }^{a, b}$, D. Bonacorsi ${ }^{a, b}$, L. Borgonovi $^{a, b}$, S. Braibant-Giacomelli $^{a, b}$,

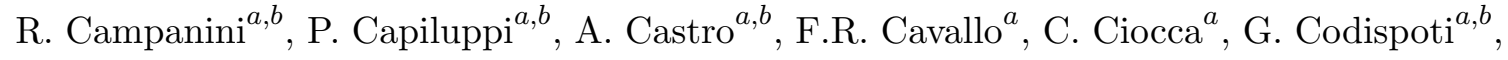


M. Cuffiani ${ }^{a, b}$, G.M. Dallavalle ${ }^{a}$, F. Fabbri ${ }^{a}$, A. Fanfani ${ }^{a, b}$, E. Fontanesi, P. Giacomelli ${ }^{a}$, C. Grandi ${ }^{a}$, L. Guiducci ${ }^{a, b}$, F. Iemmi ${ }^{a, b}$, S. Lo Meo ${ }^{a, 29}$, S. Marcellini ${ }^{a}$, G. Masetti ${ }^{a}$,

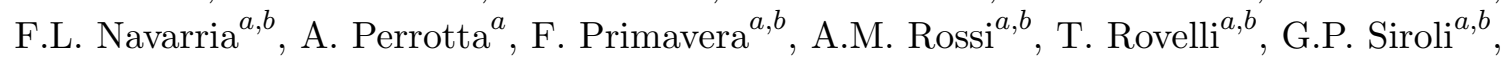
N. $\operatorname{Tosi}^{a}$

INFN Sezione di Catania ${ }^{a}$, Università di Catania ${ }^{b}$, Catania, Italy

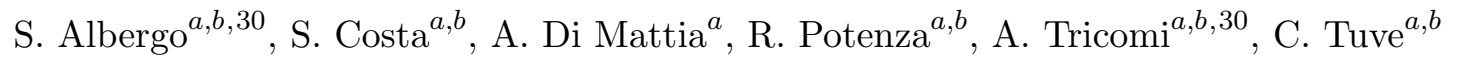

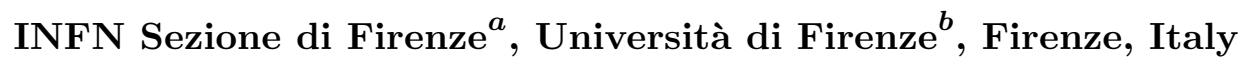

G. Barbagli ${ }^{a}$, R. Ceccarelli, K. Chatterjee ${ }^{a, b}$, V. Ciulli ${ }^{a, b}$, C. Civinini ${ }^{a}$, R. D'Alessandro ${ }^{a, b}$, E. Focardi ${ }^{a, b}$, G. Latino, P. Lenzi ${ }^{a, b}$, M. Meschini ${ }^{a}$, S. Paoletti $^{a}$, G. Sguazzoni ${ }^{a}$, D. $\operatorname{Strom}^{a}$, L. Viliani ${ }^{a}$

INFN Laboratori Nazionali di Frascati, Frascati, Italy

L. Benussi, S. Bianco, D. Piccolo

INFN Sezione di Genova ${ }^{a}$, Università di Genova ${ }^{b}$, Genova, Italy

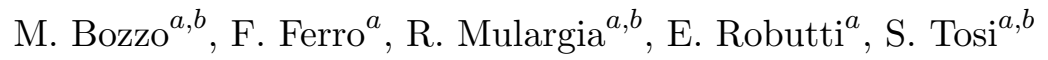

INFN Sezione di Milano-Bicocca ${ }^{a}$, Università di Milano-Bicocca ${ }^{b}$, Milano, Italy

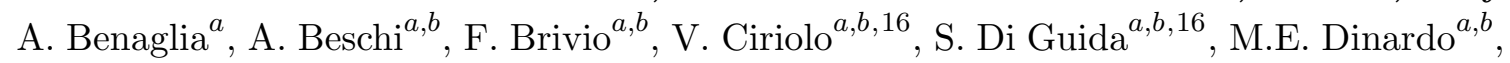
P. Dini ${ }^{a}$, S. Fiorendi ${ }^{a, b}$, S. Gennai ${ }^{a}$, A. Ghezzi ${ }^{a, b}$, P. Govoni ${ }^{a, b}$, L. Guzzi ${ }^{a, b}$, M. Malberti ${ }^{a}$, S. Malvezzi ${ }^{a}$, D. Menasce $^{a}$, F. Monti ${ }^{a, b}$, L. Moroni ${ }^{a}$, G. Ortona ${ }^{a, b}$, M. Paganoni ${ }^{a, b}$, D. Pedrini ${ }^{a}$, S. Ragazzi ${ }^{a, b}$, T. Tabarelli de Fatis ${ }^{a, b}$, D. Zuolo ${ }^{a, b}$

INFN Sezione di Napoli ${ }^{a}$, Università di Napoli 'Federico II'b , Napoli, Italy, Università della Basilicata $^{c}$, Potenza, Italy, Università G. Marconi ${ }^{d}$, Roma, Italy

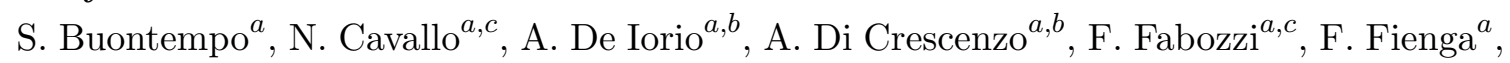
G. Galati ${ }^{a}$, A.O.M. Iorio ${ }^{a, b}$, L. Lista ${ }^{a, b}$, S. Meola ${ }^{a, d, 16}$, P. Paolucci ${ }^{a, 16}$, B. Rossi ${ }^{a}$, C. Sciacca ${ }^{a, b}$, E. Voevodina ${ }^{a, b}$

INFN Sezione di Padova ${ }^{a}$, Università di Padova ${ }^{b}$, Padova, Italy, Università di Trento ${ }^{c}$, Trento, Italy

P. Azzi ${ }^{a}$, N. Bacchetta ${ }^{a}$, D. Bisello ${ }^{a, b}$, A. Boletti ${ }^{a, b}$, A. Bragagnolo, R. Carlin ${ }^{a, b}$, P. Checchia ${ }^{a}$, P. De Castro Manzano ${ }^{a}$, T. Dorigo ${ }^{a}$, U. Dosselli ${ }^{a}$, F. Gasparini ${ }^{a, b}$, U. Gasparini $^{a, b}$, A. Gozzelino ${ }^{a}$, S.Y. Hoh, P. Lujan, M. Margoni ${ }^{a, b}$, A.T. Meneguzzo ${ }^{a, b}$,

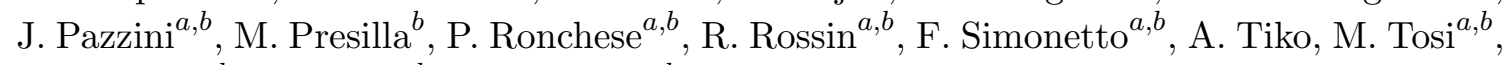
M. Zanetti ${ }^{a, b}$, P. Zotto ${ }^{a, b}$, G. Zumerle ${ }^{a, b}$

INFN Sezione di Pavia ${ }^{a}$, Università di Pavia ${ }^{b}$, Pavia, Italy

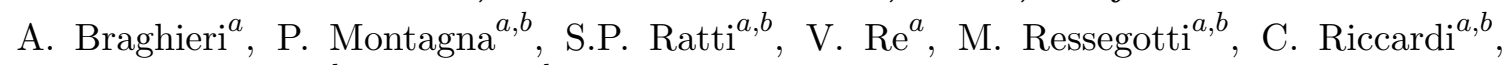
P. Salvini ${ }^{a}$, I. Vai ${ }^{a, b}$, P. Vitulo ${ }^{a, b}$

INFN Sezione di Perugia ${ }^{a}$, Università di Perugia ${ }^{b}$, Perugia, Italy

M. Biasini ${ }^{a, b}$, G.M. Bilei ${ }^{a}$, C. Cecchi ${ }^{a, b}$, D. Ciangottini ${ }^{a, b}$, L. Fanò ${ }^{a, b}$, P. Lariccia $^{a, b}$, R. Leonardi ${ }^{a, b}$, E. Manoni ${ }^{a}$, G. Mantovani ${ }^{a, b}$, V. Mariani ${ }^{a, b}$, M. Menichelli $^{a}$, A. Rossi ${ }^{a, b}$, A. Santocchia ${ }^{a, b}$, D. Spiga ${ }^{a}$ 
INFN Sezione di Pisa ${ }^{a}$, Università di Pisa ${ }^{b}$, Scuola Normale Superiore di Pisa ${ }^{c}$, Pisa, Italy

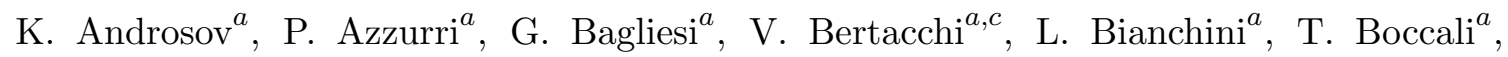

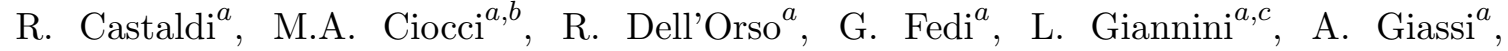
M.T. Grippo ${ }^{a}$, F. Ligabue ${ }^{a, c}$, E. Manca ${ }^{a, c}$, G. Mandorli ${ }^{a, c}$, A. Messineo ${ }^{a, b}$, F. Palla $^{a}$, A. Rizzi ${ }^{a, b}$, G. Rolandi ${ }^{31}$, S. Roy Chowdhury, A. Scribano ${ }^{a}$, P. Spagnolo $^{a}$, R. Tenchini ${ }^{a}$, G. Tonelli ${ }^{a, b}$, N. Turini, A. Venturi ${ }^{a}$, P.G. Verdini ${ }^{a}$

INFN Sezione di Roma ${ }^{a}$, Sapienza Università di Roma ${ }^{b}$, Rome, Italy

F. Cavallari ${ }^{a}$, M. Cipriani ${ }^{a, b}$, D. Del Re ${ }^{a, b}$, E. Di Marco ${ }^{a, b}$, M. Diemoz $^{a}$, E. Longo ${ }^{a, b}$,

B. Marzocchi ${ }^{a, b}$, P. Meridiani ${ }^{a}$, G. Organtini ${ }^{a, b}$, F. Pandolfi ${ }^{a}$, R. Paramatti ${ }^{a, b}$,

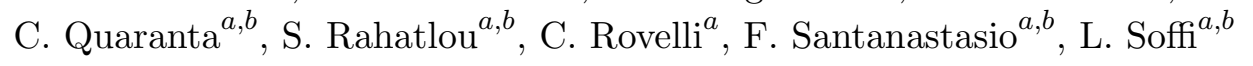

INFN Sezione di Torino ${ }^{a}$, Università di Torino ${ }^{b}$, Torino, Italy, Università del Piemonte Orientale ${ }^{c}$, Novara, Italy

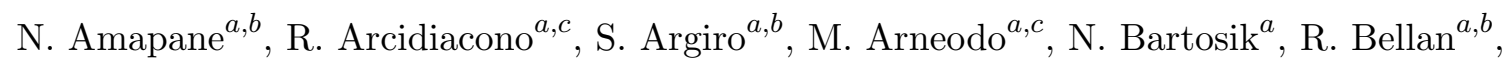
C. Biino ${ }^{a}$, A. Cappati ${ }^{a, b}$, N. Cartiglia ${ }^{a}$, S. Cometti ${ }^{a}$, M. Costa ${ }^{a, b}$, R. Covarelli ${ }^{a, b}$, N. Demaria ${ }^{a}$, B. Kiani ${ }^{a, b}$, C. Mariotti $^{a}$, S. Maselli ${ }^{a}$, E. Migliore ${ }^{a, b}$, V. Monaco ${ }^{a, b}$, E. Monteil $^{a, b}$, M. Monteno ${ }^{a}$, M.M. Obertino ${ }^{a, b}$, L. Pacher $^{a, b}$, N. Pastrone $^{a}$, M. Pelliccioni ${ }^{a}$, G.L. Pinna Angioni ${ }^{a, b}$, A. Romero ${ }^{a, b}$, M. Ruspa $^{a, c}$, R. Sacchi $^{a, b}$, R. Salvatico $^{a, b}$, V. Sola $^{a}$, A. Solano ${ }^{a, b}$, D. Soldi ${ }^{a, b}$, A. Staiano ${ }^{a}$

INFN Sezione di Trieste ${ }^{a}$, Università di Trieste ${ }^{b}$, Trieste, Italy

S. Belforte ${ }^{a}$, V. Candelise ${ }^{a, b}$, M. Casarsa ${ }^{a}$, F. Cossutti ${ }^{a}$, A. Da Rold ${ }^{a, b}$, G. Della Ricca ${ }^{a, b}$, F. Vazzoler ${ }^{a, b}$, A. Zanetti ${ }^{a}$

Kyungpook National University, Daegu, Korea

B. Kim, D.H. Kim, G.N. Kim, M.S. Kim, J. Lee, S.W. Lee, C.S. Moon, Y.D. Oh, S.I. Pak, S. Sekmen, D.C. Son, Y.C. Yang

Chonnam National University, Institute for Universe and Elementary Particles, Kwangju, Korea

H. Kim, D.H. Moon, G. Oh

Hanyang University, Seoul, Korea

B. Francois, T.J. Kim, J. Park

Korea University, Seoul, Korea

S. Cho, S. Choi, Y. Go, D. Gyun, S. Ha, B. Hong, K. Lee, K.S. Lee, J. Lim, J. Park, S.K. Park, Y. Roh

Kyung Hee University, Department of Physics

J. Goh

Sejong University, Seoul, Korea

H.S. Kim 
Seoul National University, Seoul, Korea

J. Almond, J.H. Bhyun, J. Choi, S. Jeon, J. Kim, J.S. Kim, H. Lee, K. Lee, S. Lee, K. Nam, M. Oh, S.B. Oh, B.C. Radburn-Smith, U.K. Yang, H.D. Yoo, I. Yoon, G.B. Yu

University of Seoul, Seoul, Korea

D. Jeon, H. Kim, J.H. Kim, J.S.H. Lee, I.C. Park, I. Watson

Sungkyunkwan University, Suwon, Korea

Y. Choi, C. Hwang, Y. Jeong, J. Lee, Y. Lee, I. Yu

Riga Technical University, Riga, Latvia

V. Veckalns ${ }^{32}$

Vilnius University, Vilnius, Lithuania

V. Dudenas, A. Juodagalvis, G. Tamulaitis, J. Vaitkus

National Centre for Particle Physics, Universiti Malaya, Kuala Lumpur, Malaysia

Z.A. Ibrahim, F. Mohamad Idris ${ }^{33}$, W.A.T. Wan Abdullah, M.N. Yusli, Z. Zolkapli

Universidad de Sonora (UNISON), Hermosillo, Mexico

J.F. Benitez, A. Castaneda Hernandez, J.A. Murillo Quijada, L. Valencia Palomo

Centro de Investigacion y de Estudios Avanzados del IPN, Mexico City, Mexico

H. Castilla-Valdez, E. De La Cruz-Burelo, I. Heredia-De La Cruz ${ }^{34}$, R. Lopez-Fernandez,

A. Sanchez-Hernandez

Universidad Iberoamericana, Mexico City, Mexico

S. Carrillo Moreno, C. Oropeza Barrera, M. Ramirez-Garcia, F. Vazquez Valencia

Benemerita Universidad Autonoma de Puebla, Puebla, Mexico

J. Eysermans, I. Pedraza, H.A. Salazar Ibarguen, C. Uribe Estrada

Universidad Autónoma de San Luis Potosí, San Luis Potosí, Mexico

A. Morelos Pineda

University of Montenegro, Podgorica, Montenegro

N. Raicevic

University of Auckland, Auckland, New Zealand

D. Krofcheck

University of Canterbury, Christchurch, New Zealand

S. Bheesette, P.H. Butler

National Centre for Physics, Quaid-I-Azam University, Islamabad, Pakistan

A. Ahmad, M. Ahmad, M. Gul, Q. Hassan, H.R. Hoorani, W.A. Khan, M.A. Shah, M. Shoaib, M. Waqas

AGH University of Science and Technology Faculty of Computer Science, Electronics and Telecommunications, Krakow, Poland

V. Avati, L. Grzanka, M. Malawski 
National Centre for Nuclear Research, Swierk, Poland

H. Bialkowska, M. Bluj, B. Boimska, M. Górski, M. Kazana, M. Szleper, P. Zalewski

Institute of Experimental Physics, Faculty of Physics, University of Warsaw, Warsaw, Poland

K. Bunkowski, A. Byszuk ${ }^{35}$, K. Doroba, A. Kalinowski, M. Konecki, J. Krolikowski, M. Misiura, M. Olszewski, A. Pyskir, M. Walczak

Laboratório de Instrumentação e Física Experimental de Partículas, Lisboa, Portugal

M. Araujo, P. Bargassa, D. Bastos, A. Di Francesco, P. Faccioli, B. Galinhas, M. Gallinaro, J. Hollar, N. Leonardo, J. Seixas, K. Shchelina, G. Strong, O. Toldaiev, J. Varela

Joint Institute for Nuclear Research, Dubna, Russia

V. Alexakhin, Y. Ershov, M. Gavrilenko, A. Golunov, I. Golutvin, N. Gorbounov, I. Gorbunov, V. Karjavine, V. Korenkov, A. Lanev, A. Malakhov, V. Matveev ${ }^{36,37}$, P. Moisenz, V. Palichik, V. Perelygin, M. Savina, S. Shmatov, S. Shulha, B.S. Yuldashev ${ }^{38}$, A. Zarubin

Petersburg Nuclear Physics Institute, Gatchina (St. Petersburg), Russia

L. Chtchipounov, V. Golovtsov, Y. Ivanov, V. Kim ${ }^{39}$, E. Kuznetsova ${ }^{40}$, P. Levchenko, V. Murzin, V. Oreshkin, I. Smirnov, D. Sosnov, V. Sulimov, L. Uvarov, A. Vorobyev

Institute for Nuclear Research, Moscow, Russia

Yu. Andreev, A. Dermenev, S. Gninenko, N. Golubev, A. Karneyeu, M. Kirsanov, N. Krasnikov, A. Pashenkov, D. Tlisov, A. Toropin

Institute for Theoretical and Experimental Physics named by A.I. Alikhanov of NRC 'Kurchatov Institute', Moscow, Russia

V. Epshteyn, V. Gavrilov, N. Lychkovskaya, A. Nikitenko ${ }^{41}$, V. Popov, I. Pozdnyakov, G. Safronov, A. Spiridonov, A. Stepennov, M. Toms, E. Vlasov, A. Zhokin

Moscow Institute of Physics and Technology, Moscow, Russia

T. Aushev

National Research Nuclear University 'Moscow Engineering Physics Institute' (MEPhI), Moscow, Russia

O. Bychkova, R. Chistov ${ }^{42}$, M. Danilov ${ }^{42}$, S. Polikarpov ${ }^{42}$, E. Tarkovskii

P.N. Lebedev Physical Institute, Moscow, Russia

V. Andreev, M. Azarkin, I. Dremin, M. Kirakosyan, A. Terkulov

Skobeltsyn Institute of Nuclear Physics, Lomonosov Moscow State University, Moscow, Russia

A. Baskakov, A. Belyaev, E. Boos, V. Bunichev, M. Dubinin ${ }^{43}$, L. Dudko, V. Klyukhin,

O. Kodolova, I. Lokhtin, S. Obraztsov, M. Perfilov, S. Petrushanko, V. Savrin

Novosibirsk State University (NSU), Novosibirsk, Russia

A. Barnyakov ${ }^{44}$, V. Blinov ${ }^{44}$, T. Dimova ${ }^{44}$, L. Kardapoltsev ${ }^{44}$, Y. Skovpen ${ }^{44}$ 
Institute for High Energy Physics of National Research Centre 'Kurchatov Institute', Protvino, Russia

I. Azhgirey, I. Bayshev, S. Bitioukov, V. Kachanov, D. Konstantinov, P. Mandrik, V. Petrov, R. Ryutin, S. Slabospitskii, A. Sobol, S. Troshin, N. Tyurin, A. Uzunian, A. Volkov

National Research Tomsk Polytechnic University, Tomsk, Russia

A. Babaev, A. Iuzhakov, V. Okhotnikov

Tomsk State University, Tomsk, Russia

V. Borchsh, V. Ivanchenko, E. Tcherniaev

University of Belgrade: Faculty of Physics and VINCA Institute of Nuclear Sciences

P. Adzic ${ }^{45}$, P. Cirkovic, D. Devetak, M. Dordevic, P. Milenovic, J. Milosevic, M. Stojanovic

Centro de Investigaciones Energéticas Medioambientales y Tecnológicas (CIEMAT), Madrid, Spain

M. Aguilar-Benitez, J. Alcaraz Maestre, A. Álvarez Fernández, I. Bachiller, M. Barrio Luna, J.A. Brochero Cifuentes, C.A. Carrillo Montoya, M. Cepeda, M. Cerrada, N. Colino, B. De La Cruz, A. Delgado Peris, C. Fernandez Bedoya, J.P. Fernández Ramos, J. Flix, M.C. Fouz, O. Gonzalez Lopez, S. Goy Lopez, J.M. Hernandez, M.I. Josa, D. Moran, Á. Navarro Tobar, A. Pérez-Calero Yzquierdo, J. Puerta Pelayo, I. Redondo, L. Romero, S. Sánchez Navas, M.S. Soares, A. Triossi, C. Willmott

Universidad Autónoma de Madrid, Madrid, Spain

C. Albajar, J.F. de Trocóniz

Universidad de Oviedo, Instituto Universitario de Ciencias y Tecnologías Espaciales de Asturias (ICTEA), Oviedo, Spain

B. Alvarez Gonzalez, J. Cuevas, C. Erice, J. Fernandez Menendez, S. Folgueras, I. Gonzalez Caballero, J.R. González Fernández, E. Palencia Cortezon, V. Rodríguez Bouza, S. Sanchez Cruz

Instituto de Física de Cantabria (IFCA), CSIC-Universidad de Cantabria, Santander, Spain

I.J. Cabrillo, A. Calderon, B. Chazin Quero, J. Duarte Campderros, M. Fernandez, P.J. Fernández Manteca, A. García Alonso, G. Gomez, C. Martinez Rivero, P. Martinez Ruiz del Arbol, F. Matorras, J. Piedra Gomez, C. Prieels, T. Rodrigo, A. Ruiz-Jimeno, L. Russo ${ }^{46}$, L. Scodellaro, N. Trevisani, I. Vila, J.M. Vizan Garcia

University of Colombo, Colombo, Sri Lanka

K. Malagalage

University of Ruhuna, Department of Physics, Matara, Sri Lanka

W.G.D. Dharmaratna, N. Wickramage 


\section{CERN, European Organization for Nuclear Research, Geneva, Switzerland}

D. Abbaneo, B. Akgun, E. Auffray, G. Auzinger, J. Baechler, P. Baillon, A.H. Ball, D. Barney, J. Bendavid, M. Bianco, A. Bocci, E. Bossini, C. Botta, E. Brondolin, T. Camporesi, A. Caratelli, G. Cerminara, E. Chapon, G. Cucciati, D. d'Enterria, A. Dabrowski, N. Daci, V. Daponte, A. David, O. Davignon, A. De Roeck, N. Deelen, M. Deile, M. Dobson, M. Dünser, N. Dupont, A. Elliott-Peisert, F. Fallavollita ${ }^{47}$, D. Fasanella, G. Franzoni, J. Fulcher, W. Funk, S. Giani, D. Gigi, A. Gilbert, K. Gill, F. Glege, M. Gruchala, M. Guilbaud, D. Gulhan, J. Hegeman, C. Heidegger, Y. Iiyama, V. Innocente, P. Janot, O. Karacheban ${ }^{19}$, J. Kaspar, J. Kieseler, M. Krammer ${ }^{1}$, C. Lange, P. Lecoq, C. Lourenço, L. Malgeri, M. Mannelli, A. Massironi, F. Meijers, J.A. Merlin, S. Mersi, E. Meschi, F. Moortgat, M. Mulders, J. Ngadiuba, S. Nourbakhsh, S. Orfanelli, L. Orsini, F. Pantaleo ${ }^{16}$, L. Pape, E. Perez, M. Peruzzi, A. Petrilli, G. Petrucciani, A. Pfeiffer, M. Pierini, F.M. Pitters, D. Rabady, A. Racz, M. Rovere, H. Sakulin, C. Schäfer, C. Schwick, M. Selvaggi, A. Sharma, P. Silva, W. Snoeys, P. Sphicas ${ }^{48}$, J. Steggemann, V.R. Tavolaro, D. Treille, A. Tsirou, A. Vartak, M. Verzetti, W.D. Zeuner

\section{Paul Scherrer Institut, Villigen, Switzerland}

L. Caminada ${ }^{49}$, K. Deiters, W. Erdmann, R. Horisberger, Q. Ingram, H.C. Kaestli, D. Kotlinski, U. Langenegger, T. Rohe, S.A. Wiederkehr

ETH Zurich - Institute for Particle Physics and Astrophysics (IPA), Zurich, Switzerland

M. Backhaus, P. Berger, N. Chernyavskaya, G. Dissertori, M. Dittmar, M. Donegà, C. Dorfer, T.A. Gómez Espinosa, C. Grab, D. Hits, T. Klijnsma, W. Lustermann, R.A. Manzoni, M. Marionneau, M.T. Meinhard, F. Micheli, P. Musella, F. Nessi-Tedaldi, F. Pauss, G. Perrin, L. Perrozzi, S. Pigazzini, M. Reichmann, C. Reissel, T. Reitenspiess, D. Ruini, D.A. Sanz Becerra, M. Schönenberger, L. Shchutska, M.L. Vesterbacka Olsson, R. Wallny, D.H. Zhu

\section{Universität Zürich, Zurich, Switzerland}

T.K. Aarrestad, C. Amsler ${ }^{50}$, D. Brzhechko, M.F. Canelli, A. De Cosa, R. Del Burgo, S. Donato, B. Kilminster, S. Leontsinis, V.M. Mikuni, I. Neutelings, G. Rauco, P. Robmann, D. Salerno, K. Schweiger, C. Seitz, Y. Takahashi, S. Wertz, A. Zucchetta

\section{National Central University, Chung-Li, Taiwan}

T.H. Doan, C.M. Kuo, W. Lin, A. Roy, S.S. Yu

\section{National Taiwan University (NTU), Taipei, Taiwan}

P. Chang, Y. Chao, K.F. Chen, P.H. Chen, W.-S. Hou, Y.y. Li, R.-S. Lu, E. Paganis, A. Psallidas, A. Steen

Chulalongkorn University, Faculty of Science, Department of Physics, Bangkok, Thailand

B. Asavapibhop, C. Asawatangtrakuldee, N. Srimanobhas, N. Suwonjandee 
Çukurova University, Physics Department, Science and Art Faculty, Adana, Turkey

A. Bat, F. Boran, S. Damarseckin ${ }^{51}$, Z.S. Demiroglu, F. Dolek, C. Dozen, I. Dumanoglu, E. Eskut, G. Gokbulut, EmineGurpinar Guler ${ }^{52}$, Y. Guler, I. Hos ${ }^{53}$, C. Isik, E.E. Kangal ${ }^{54}$, O. Kara, A. Kayis Topaksu, U. Kiminsu, M. Oglakci, G. Onengut, K. Ozdemir ${ }^{55}$, A. Polatoz, A.E. Simsek, D. Sunar Cerci ${ }^{56}$, U.G. Tok, S. Turkcapar, I.S. Zorbakir, C. Zorbilmez

Middle East Technical University, Physics Department, Ankara, Turkey

B. Isildak ${ }^{57}$, G. Karapinar ${ }^{58}$, M. Yalvac

Bogazici University, Istanbul, Turkey

I.O. Atakisi, E. Gülmez, M. Kaya ${ }^{59}$, O. Kaya ${ }^{60}$, B. Kaynak, Ö. Özçelik, S. Tekten, E.A. Yetkin ${ }^{61}$

Istanbul Technical University, Istanbul, Turkey

A. Cakir, K. Cankocak, Y. Komurcu, S. Sen ${ }^{62}$

Istanbul University, Istanbul, Turkey

S. Ozkorucuklu

Institute for Scintillation Materials of National Academy of Science of Ukraine, Kharkov, Ukraine

B. Grynyov

National Scientific Center, Kharkov Institute of Physics and Technology, Kharkov, Ukraine

L. Levchuk

University of Bristol, Bristol, United Kingdom

F. Ball, E. Bhal, S. Bologna, J.J. Brooke, D. Burns, E. Clement, D. Cussans, H. Flacher, J. Goldstein, G.P. Heath, H.F. Heath, L. Kreczko, S. Paramesvaran, B. Penning, T. Sakuma, S. Seif El Nasr-Storey, D. Smith, V.J. Smith, J. Taylor, A. Titterton

Rutherford Appleton Laboratory, Didcot, United Kingdom

K.W. Bell, A. Belyaev ${ }^{63}$, C. Brew, R.M. Brown, D. Cieri, D.J.A. Cockerill, J.A. Coughlan, K. Harder, S. Harper, J. Linacre, K. Manolopoulos, D.M. Newbold, E. Olaiya, D. Petyt, T. Reis, T. Schuh, C.H. Shepherd-Themistocleous, A. Thea, I.R. Tomalin, T. Williams, W.J. Womersley

\section{Imperial College, London, United Kingdom}

R. Bainbridge, P. Bloch, J. Borg, S. Breeze, O. Buchmuller, A. Bundock, GurpreetSingh CHAHAL ${ }^{64}$, D. Colling, P. Dauncey, G. Davies, M. Della Negra, R. Di Maria, P. Everaerts, G. Hall, G. Iles, T. James, M. Komm, C. Laner, L. Lyons, A.-M. Magnan, S. Malik, A. Martelli, V. Milosevic, J. $\mathrm{Nash}^{65}$, V. Palladino, M. Pesaresi, D.M. Raymond, A. Richards, A. Rose, E. Scott, C. Seez, A. Shtipliyski, M. Stoye, T. Strebler, S. Summers, A. Tapper, K. Uchida, T. Virdee ${ }^{16}$, N. Wardle, D. Winterbottom, J. Wright, A.G. Zecchinelli, S.C. Zenz 
Brunel University, Uxbridge, United Kingdom

J.E. Cole, P.R. Hobson, A. Khan, P. Kyberd, C.K. Mackay, A. Morton, I.D. Reid, L. Teodorescu, S. Zahid

Baylor University, Waco, U.S.A.

K. Call, J. Dittmann, K. Hatakeyama, C. Madrid, B. McMaster, N. Pastika, C. Smith

Catholic University of America, Washington, DC, U.S.A.

R. Bartek, A. Dominguez, R. Uniyal

The University of Alabama, Tuscaloosa, U.S.A.

A. Buccilli, S.I. Cooper, C. Henderson, P. Rumerio, C. West

Boston University, Boston, U.S.A.

D. Arcaro, T. Bose, Z. Demiragli, D. Gastler, S. Girgis, D. Pinna, C. Richardson, J. Rohlf,

D. Sperka, I. Suarez, L. Sulak, D. Zou

Brown University, Providence, U.S.A.

G. Benelli, B. Burkle, X. Coubez, D. Cutts, Y.t. Duh, M. Hadley, J. Hakala, U. Heintz, J.M. $\operatorname{Hogan}^{66}$, K.H.M. Kwok, E. Laird, G. Landsberg, J. Lee, Z. Mao, M. Narain, S. Sagir ${ }^{67}$, R. Syarif, E. Usai, D. Yu

University of California, Davis, Davis, U.S.A.

R. Band, C. Brainerd, R. Breedon, M. Calderon De La Barca Sanchez, M. Chertok, J. Conway, R. Conway, P.T. Cox, R. Erbacher, C. Flores, G. Funk, F. Jensen, W. Ko, O. Kukral, R. Lander, M. Mulhearn, D. Pellett, J. Pilot, M. Shi, D. Stolp, D. Taylor, K. Tos, M. Tripathi, Z. Wang, F. Zhang

University of California, Los Angeles, U.S.A.

M. Bachtis, C. Bravo, R. Cousins, A. Dasgupta, A. Florent, J. Hauser, M. Ignatenko, N. Mccoll, W.A. Nash, S. Regnard, D. Saltzberg, C. Schnaible, B. Stone, V. Valuev

University of California, Riverside, Riverside, U.S.A.

K. Burt, R. Clare, J.W. Gary, S.M.A. Ghiasi Shirazi, G. Hanson, G. Karapostoli, E. Kennedy, O.R. Long, M. Olmedo Negrete, M.I. Paneva, W. Si, L. Wang, H. Wei, S. Wimpenny, B.R. Yates, Y. Zhang

University of California, San Diego, La Jolla, U.S.A.

J.G. Branson, P. Chang, S. Cittolin, M. Derdzinski, R. Gerosa, D. Gilbert, B. Hashemi, D. Klein, V. Krutelyov, J. Letts, M. Masciovecchio, S. May, S. Padhi, M. Pieri, V. Sharma, M. Tadel, F. Würthwein, A. Yagil, G. Zevi Della Porta

University of California, Santa Barbara - Department of Physics, Santa Barbara, U.S.A.

N. Amin, R. Bhandari, C. Campagnari, M. Citron, V. Dutta, M. Franco Sevilla, L. Gouskos, J. Incandela, B. Marsh, H. Mei, A. Ovcharova, H. Qu, J. Richman, U. Sarica, D. Stuart, S. Wang, J. Yoo 
California Institute of Technology, Pasadena, U.S.A.

D. Anderson, A. Bornheim, O. Cerri, I. Dutta, J.M. Lawhorn, N. Lu, J. Mao, H.B. Newman, T.Q. Nguyen, J. Pata, M. Spiropulu, J.R. Vlimant, S. Xie, Z. Zhang, R.Y. Zhu

Carnegie Mellon University, Pittsburgh, U.S.A.

M.B. Andrews, T. Ferguson, T. Mudholkar, M. Paulini, M. Sun, I. Vorobiev, M. Weinberg

University of Colorado Boulder, Boulder, U.S.A.

J.P. Cumalat, W.T. Ford, A. Johnson, E. MacDonald, T. Mulholland, R. Patel, A. Perloff, K. Stenson, K.A. Ulmer, S.R. Wagner

Cornell University, Ithaca, U.S.A.

J. Alexander, J. Chaves, Y. Cheng, J. Chu, A. Datta, A. Frankenthal, K. Mcdermott, N. Mirman, J.R. Patterson, D. Quach, A. Rinkevicius ${ }^{68}$, A. Ryd, S.M. Tan, Z. Tao, J. Thom, P. Wittich, M. Zientek

Fermi National Accelerator Laboratory, Batavia, U.S.A.

S. Abdullin, M. Albrow, M. Alyari, G. Apollinari, A. Apresyan, A. Apyan, S. Banerjee, L.A.T. Bauerdick, A. Beretvas, J. Berryhill, P.C. Bhat, K. Burkett, J.N. Butler, A. Canepa, G.B. Cerati, H.W.K. Cheung, F. Chlebana, M. Cremonesi, J. Duarte, V.D. Elvira, J. Freeman, Z. Gecse, E. Gottschalk, L. Gray, D. Green, S. Grünendahl, O. Gutsche, AllisonReinsvold Hall, J. Hanlon, R.M. Harris, S. Hasegawa, R. Heller, J. Hirschauer, B. Jayatilaka, S. Jindariani, M. Johnson, U. Joshi, B. Klima, M.J. Kortelainen, B. Kreis, S. Lammel, J. Lewis, D. Lincoln, R. Lipton, M. Liu, T. Liu, J. Lykken, K. Maeshima, J.M. Marraffino, D. Mason, P. McBride, P. Merkel, S. Mrenna, S. Nahn, V. O’Dell, V. Papadimitriou, K. Pedro, C. Pena, G. Rakness, F. Ravera, L. Ristori, B. Schneider,

E. Sexton-Kennedy, N. Smith, A. Soha, W.J. Spalding, L. Spiegel, S. Stoynev, J. Strait, N. Strobbe, L. Taylor, S. Tkaczyk, N.V. Tran, L. Uplegger, E.W. Vaandering, C. Vernieri, M. Verzocchi, R. Vidal, M. Wang, H.A. Weber

University of Florida, Gainesville, U.S.A.

D. Acosta, P. Avery, P. Bortignon, D. Bourilkov, A. Brinkerhoff, L. Cadamuro, A. Carnes, V. Cherepanov, D. Curry, F. Errico, R.D. Field, S.V. Gleyzer, B.M. Joshi, M. Kim, J. Konigsberg, A. Korytov, K.H. Lo, P. Ma, K. Matchev, N. Menendez, G. Mitselmakher, D. Rosenzweig, K. Shi, J. Wang, S. Wang, X. Zuo

Florida International University, Miami, U.S.A.

Y.R. Joshi

Florida State University, Tallahassee, U.S.A.

T. Adams, A. Askew, S. Hagopian, V. Hagopian, K.F. Johnson, R. Khurana, T. Kolberg, G. Martinez, T. Perry, H. Prosper, C. Schiber, R. Yohay, J. Zhang

Florida Institute of Technology, Melbourne, U.S.A.

M.M. Baarmand, V. Bhopatkar, M. Hohlmann, D. Noonan, M. Rahmani, M. Saunders, F. Yumiceva 
University of Illinois at Chicago (UIC), Chicago, U.S.A.

M.R. Adams, L. Apanasevich, D. Berry, R.R. Betts, R. Cavanaugh, X. Chen, S. Dittmer, O. Evdokimov, C.E. Gerber, D.A. Hangal, D.J. Hofman, K. Jung, C. Mills, T. Roy, M.B. Tonjes, N. Varelas, H. Wang, X. Wang, Z. Wu

The University of Iowa, Iowa City, U.S.A.

M. Alhusseini, B. Bilki ${ }^{52}$, W. Clarida, K. Dilsiz ${ }^{69}$, S. Durgut, R.P. Gandrajula, M. Haytmyradov, V. Khristenko, O.K. Köseyan, J.-P. Merlo, A. Mestvirishvili ${ }^{70}$, A. Moeller, J. Nachtman, H. Ogul ${ }^{71}$, Y. Onel, F. Ozok ${ }^{72}$, A. Penzo, C. Snyder, E. Tiras, J. Wetzel

Johns Hopkins University, Baltimore, U.S.A.

B. Blumenfeld, A. Cocoros, N. Eminizer, D. Fehling, L. Feng, A.V. Gritsan, W.T. Hung, P. Maksimovic, J. Roskes, M. Swartz, M. Xiao

The University of Kansas, Lawrence, U.S.A.

C. Baldenegro Barrera, P. Baringer, A. Bean, S. Boren, J. Bowen, A. Bylinkin, T. Isidori, S. Khalil, J. King, G. Krintiras, A. Kropivnitskaya, C. Lindsey, D. Majumder, W. Mcbrayer, N. Minafra, M. Murray, C. Rogan, C. Royon, S. Sanders, E. Schmitz, J.D. Tapia Takaki, Q. Wang, J. Williams, G. Wilson

Kansas State University, Manhattan, U.S.A.

S. Duric, A. Ivanov, K. Kaadze, D. Kim, Y. Maravin, D.R. Mendis, T. Mitchell, A. Modak, A. Mohammadi

Lawrence Livermore National Laboratory, Livermore, U.S.A.

F. Rebassoo, D. Wright

University of Maryland, College Park, U.S.A.

A. Baden, O. Baron, A. Belloni, S.C. Eno, Y. Feng, N.J. Hadley, S. Jabeen, G.Y. Jeng, R.G. Kellogg, J. Kunkle, A.C. Mignerey, S. Nabili, F. Ricci-Tam, M. Seidel, Y.H. Shin, A. Skuja, S.C. Tonwar, K. Wong

Massachusetts Institute of Technology, Cambridge, U.S.A.

D. Abercrombie, B. Allen, A. Baty, R. Bi, S. Brandt, W. Busza, I.A. Cali, M. D'Alfonso,

G. Gomez Ceballos, M. Goncharov, P. Harris, D. Hsu, M. Hu, M. Klute, D. Kovalskyi, Y.-J. Lee, P.D. Luckey, B. Maier, A.C. Marini, C. Mcginn, C. Mironov, S. Narayanan, X. Niu, C. Paus, D. Rankin, C. Roland, G. Roland, Z. Shi, G.S.F. Stephans, K. Sumorok, K. Tatar, D. Velicanu, J. Wang, T.W. Wang, B. Wyslouch

University of Minnesota, Minneapolis, U.S.A.

A.C. Benvenuti ${ }^{\dagger}$, R.M. Chatterjee, A. Evans, S. Guts, P. Hansen, J. Hiltbrand, Sh. Jain, S. Kalafut, Y. Kubota, Z. Lesko, J. Mans, R. Rusack, M.A. Wadud

University of Mississippi, Oxford, U.S.A.

J.G. Acosta, S. Oliveros

University of Nebraska-Lincoln, Lincoln, U.S.A.

K. Bloom, D.R. Claes, C. Fangmeier, L. Finco, F. Golf, R. Gonzalez Suarez, R. Kamalieddin, I. Kravchenko, J.E. Siado, G.R. Snow, B. Stieger 
State University of New York at Buffalo, Buffalo, U.S.A.

G. Agarwal, C. Harrington, I. Iashvili, A. Kharchilava, C. Mclean, D. Nguyen, A. Parker, J. Pekkanen, S. Rappoccio, B. Roozbahani

Northeastern University, Boston, U.S.A.

G. Alverson, E. Barberis, C. Freer, Y. Haddad, A. Hortiangtham, G. Madigan, D.M. Morse,

T. Orimoto, L. Skinnari, A. Tishelman-Charny, T. Wamorkar, B. Wang, A. Wisecarver,

D. Wood

Northwestern University, Evanston, U.S.A.

S. Bhattacharya, J. Bueghly, T. Gunter, K.A. Hahn, N. Odell, M.H. Schmitt, K. Sung, M. Trovato, M. Velasco

University of Notre Dame, Notre Dame, U.S.A.

R. Bucci, N. Dev, R. Goldouzian, M. Hildreth, K. Hurtado Anampa, C. Jessop, D.J. Karmgard, K. Lannon, W. Li, N. Loukas, N. Marinelli, I. Mcalister, F. Meng, C. Mueller, Y. Musienko ${ }^{36}$, M. Planer, R. Ruchti, P. Siddireddy, G. Smith, S. Taroni, M. Wayne, A. Wightman, M. Wolf, A. Woodard

The Ohio State University, Columbus, U.S.A.

J. Alimena, B. Bylsma, L.S. Durkin, S. Flowers, B. Francis, C. Hill, W. Ji, A. Lefeld, T.Y. Ling, B.L. Winer

Princeton University, Princeton, U.S.A.

S. Cooperstein, G. Dezoort, P. Elmer, J. Hardenbrook, N. Haubrich, S. Higginbotham, A. Kalogeropoulos, S. Kwan, D. Lange, M.T. Lucchini, J. Luo, D. Marlow, K. Mei, I. Ojalvo, J. Olsen, C. Palmer, P. Piroué, J. Salfeld-Nebgen, D. Stickland, C. Tully, Z. Wang

University of Puerto Rico, Mayaguez, U.S.A.

S. Malik, S. Norberg

Purdue University, West Lafayette, U.S.A.

A. Barker, V.E. Barnes, S. Das, L. Gutay, M. Jones, A.W. Jung, A. Khatiwada, B. Mahakud, D.H. Miller, G. Negro, N. Neumeister, C.C. Peng, S. Piperov, H. Qiu, J.F. Schulte, J. Sun, F. Wang, R. Xiao, W. Xie

Purdue University Northwest, Hammond, U.S.A.

T. Cheng, J. Dolen, N. Parashar

Rice University, Houston, U.S.A.

K.M. Ecklund, S. Freed, F.J.M. Geurts, M. Kilpatrick, Arun Kumar, W. Li, B.P. Padley, R. Redjimi, J. Roberts, J. Rorie, W. Shi, A.G. Stahl Leiton, Z. Tu, A. Zhang

University of Rochester, Rochester, U.S.A.

A. Bodek, P. de Barbaro, R. Demina, J.L. Dulemba, C. Fallon, T. Ferbel, M. Galanti, A. Garcia-Bellido, J. Han, O. Hindrichs, A. Khukhunaishvili, E. Ranken, P. Tan, R. Taus 
Rutgers, The State University of New Jersey, Piscataway, U.S.A.

B. Chiarito, J.P. Chou, A. Gandrakota, Y. Gershtein, E. Halkiadakis, A. Hart, M. Heindl, E. Hughes, S. Kaplan, S. Kyriacou, I. Laflotte, A. Lath, R. Montalvo, K. Nash, M. Osherson, H. Saka, S. Salur, S. Schnetzer, D. Sheffield, S. Somalwar, R. Stone, S. Thomas, P. Thomassen

University of Tennessee, Knoxville, U.S.A.

H. Acharya, A.G. Delannoy, J. Heideman, G. Riley, S. Spanier

Texas A\&M University, College Station, U.S.A.

O. Bouhali ${ }^{73}$, A. Celik, M. Dalchenko, M. De Mattia, A. Delgado, S. Dildick, R. Eusebi, J. Gilmore, T. Huang, T. Kamon ${ }^{74}$, S. Luo, D. Marley, R. Mueller, D. Overton, L. Perniè, D. Rathjens, A. Safonov

\section{Texas Tech University, Lubbock, U.S.A.}

N. Akchurin, J. Damgov, F. De Guio, S. Kunori, K. Lamichhane, S.W. Lee, T. Mengke, S. Muthumuni, T. Peltola, S. Undleeb, I. Volobouev, Z. Wang, A. Whitbeck

Vanderbilt University, Nashville, U.S.A.

S. Greene, A. Gurrola, R. Janjam, W. Johns, C. Maguire, A. Melo, H. Ni, K. Padeken, F. Romeo, P. Sheldon, S. Tuo, J. Velkovska, M. Verweij

University of Virginia, Charlottesville, U.S.A.

M.W. Arenton, P. Barria, B. Cox, G. Cummings, R. Hirosky, M. Joyce, A. Ledovskoy, C. Neu, B. Tannenwald, Y. Wang, E. Wolfe, F. Xia

\section{Wayne State University, Detroit, U.S.A.}

R. Harr, P.E. Karchin, N. Poudyal, J. Sturdy, P. Thapa, S. Zaleski

University of Wisconsin - Madison, Madison, WI, U.S.A.

J. Buchanan, C. Caillol, D. Carlsmith, S. Dasu, I. De Bruyn, L. Dodd, F. Fiori, C. Galloni, B. Gomber ${ }^{75}$, M. Herndon, A. Hervé, U. Hussain, P. Klabbers, A. Lanaro, A. Loeliger, K. Long, R. Loveless, J. Madhusudanan Sreekala, T. Ruggles, A. Savin, V. Sharma, W.H. Smith, D. Teague, S. Trembath-reichert, N. Woods

$\dagger$ : Deceased

1: Also at Vienna University of Technology, Vienna, Austria

2: Also at IRFU, CEA, Université Paris-Saclay, Gif-sur-Yvette, France

3: Also at Universidade Estadual de Campinas, Campinas, Brazil

4: Also at Federal University of Rio Grande do Sul, Porto Alegre, Brazil

5: Also at UFMS, Nova Andradina, Brazil

6: Also at Universidade Federal de Pelotas, Pelotas, Brazil

7: Also at Université Libre de Bruxelles, Bruxelles, Belgium

8: Also at University of Chinese Academy of Sciences, Beijing, China

9: Also at Institute for Theoretical and Experimental Physics named by A.I. Alikhanov of NRC 'Kurchatov Institute', Moscow, Russia

10: Also at Joint Institute for Nuclear Research, Dubna, Russia

11: Also at Cairo University, Cairo, Egypt 
12: Also at Zewail City of Science and Technology, Zewail, Egypt

13: Also at Purdue University, West Lafayette, U.S.A.

14: Also at Université de Haute Alsace, Mulhouse, France

15: Also at Erzincan Binali Yildirim University, Erzincan, Turkey

16: Also at CERN, European Organization for Nuclear Research, Geneva, Switzerland

17: Also at RWTH Aachen University, III. Physikalisches Institut A, Aachen, Germany

18: Also at University of Hamburg, Hamburg, Germany

19: Also at Brandenburg University of Technology, Cottbus, Germany

20: Also at Institute of Physics, University of Debrecen, Debrecen, Hungary, Debrecen, Hungary

21: Also at Institute of Nuclear Research ATOMKI, Debrecen, Hungary

22: Also at MTA-ELTE Lendület CMS Particle and Nuclear Physics Group, Eötvös Loránd University, Budapest, Hungary, Budapest, Hungary

23: Also at IIT Bhubaneswar, Bhubaneswar, India, Bhubaneswar, India

24: Also at Institute of Physics, Bhubaneswar, India

25: Also at Shoolini University, Solan, India

26: Also at University of Visva-Bharati, Santiniketan, India

27: Also at Isfahan University of Technology, Isfahan, Iran

28: Now at INFN Sezione di Bari ${ }^{a}$, Università di Bari ${ }^{b}$, Politecnico di Bari ${ }^{c}$, Bari, Italy

29: Also at Italian National Agency for New Technologies, Energy and Sustainable Economic Development, Bologna, Italy

30: Also at Centro Siciliano di Fisica Nucleare e di Struttura Della Materia, Catania, Italy

31: Also at Scuola Normale e Sezione dell'INFN, Pisa, Italy

32: Also at Riga Technical University, Riga, Latvia, Riga, Latvia

33: Also at Malaysian Nuclear Agency, MOSTI, Kajang, Malaysia

34: Also at Consejo Nacional de Ciencia y Tecnología, Mexico City, Mexico

35: Also at Warsaw University of Technology, Institute of Electronic Systems, Warsaw, Poland

36: Also at Institute for Nuclear Research, Moscow, Russia

37: Now at National Research Nuclear University 'Moscow Engineering Physics Institute' (MEPhI), Moscow, Russia

38: Also at Institute of Nuclear Physics of the Uzbekistan Academy of Sciences, Tashkent, Uzbekistan

39: Also at St. Petersburg State Polytechnical University, St. Petersburg, Russia

40: Also at University of Florida, Gainesville, U.S.A.

41: Also at Imperial College, London, United Kingdom

42: Also at P.N. Lebedev Physical Institute, Moscow, Russia

43: Also at California Institute of Technology, Pasadena, U.S.A.

44: Also at Budker Institute of Nuclear Physics, Novosibirsk, Russia

45: Also at Faculty of Physics, University of Belgrade, Belgrade, Serbia

46: Also at Università degli Studi di Siena, Siena, Italy

47: Also at INFN Sezione di Pavia ${ }^{a}$, Università di Pavia ${ }^{b}$, Pavia, Italy, Pavia, Italy

48: Also at National and Kapodistrian University of Athens, Athens, Greece

49: Also at Universität Zürich, Zurich, Switzerland

50: Also at Stefan Meyer Institute for Subatomic Physics, Vienna, Austria, Vienna, Austria

51: Also at Şırnak University, Sirnak, Turkey

52: Also at Beykent University, Istanbul, Turkey, Istanbul, Turkey

53: Also at Istanbul Aydin University, Application and Research Center for Advanced Studies (App. \& Res. Cent. for Advanced Studies), Istanbul, Turkey

54: Also at Mersin University, Mersin, Turkey 
55: Also at Piri Reis University, Istanbul, Turkey

56: Also at Adiyaman University, Adiyaman, Turkey

57: Also at Ozyegin University, Istanbul, Turkey

58: Also at Izmir Institute of Technology, Izmir, Turkey

59: Also at Marmara University, Istanbul, Turkey

60: Also at Kafkas University, Kars, Turkey

61: Also at Istanbul Bilgi University, Istanbul, Turkey

62: Also at Hacettepe University, Ankara, Turkey

63: Also at School of Physics and Astronomy, University of Southampton, Southampton, United Kingdom

64: Also at IPPP Durham University, Durham, United Kingdom

65: Also at Monash University, Faculty of Science, Clayton, Australia

66: Also at Bethel University, St. Paul, Minneapolis, U.S.A., St. Paul, U.S.A.

67: Also at Karamanoğlu Mehmetbey University, Karaman, Turkey

68: Also at Vilnius University, Vilnius, Lithuania

69: Also at Bingol University, Bingol, Turkey

70: Also at Georgian Technical University, Tbilisi, Georgia

71: Also at Sinop University, Sinop, Turkey

72: Also at Mimar Sinan University, Istanbul, Istanbul, Turkey

73: Also at Texas A\&M University at Qatar, Doha, Qatar

74: Also at Kyungpook National University, Daegu, Korea, Daegu, Korea

75: Also at University of Hyderabad, Hyderabad, India 